\title{
Deviation of a Monochromatic Lamb Wave Beam in Anisotropic Multilayered Media: Asymptotic Analysis, Numerical and Experimental Results
}

\author{
Catherine Potel, Stéphane Baly, Jean-François de Belleval, Member, IEEE, \\ Mike Lowe, and Philippe Gatignol
}

\begin{abstract}
The aim of this paper is threefold: to describe the physical phenomenon of the excitation of modal waves such as Lamb waves, in anisotropic multilayered media, by a monochromatic incident beam, using an asymptotic approach; to present a three-dimensional model using the decomposition of the incident beam into monochromatic plane waves (the formalism is applied to the particle displacement vector); to illustrate the phenomenon both numerically and experimentally. Numerical and experimental maps of the reflected field of pressure are presented, and the reradiation of the Lamb wave beam in an oblique plane is theoretically and numerically illustrated.
\end{abstract}

\section{INTRODUCTION}

$\mathrm{T}$ HE ultrasonic testing of anisotropic multilayered structures is a well developed field of research, motivated strongly by the needs for inspection of carbon fiber composite materials for the aircraft industry. The challenges include the measurement of the mechanical properties of the structures to ensure manufacture quality, the detection of defects that may be created accidentally during manufacture, and the detection of defects that may be introduced in service. Recently, interest in the concept of structural health monitoring has come to the forefront, and the challenge here is to monitor large areas of composite structures using a limited number of permanently attached transducers. A very interesting approach for these kinds of inspection is the application of structure-guided waves, such as Lamb waves, which enable a whole strip to be tested with a single shot. However, the understanding of the interaction of ultrasonic waves with anisotropic multilayered structures is not easily achieved, and a solid base of theoretical work is essential for the effective development of testing techniques. Numerous works that ad-

Manuscript received April 15, 2004; accepted December 21, 2004. C. Potel is with Laboratoire d'Acoustique de l'Université du Maine (LAUM), UMR CNRS 6613, Université du Maine, Avenue Olivier Messiaen, 72085 Le Mans, Cedex 9, France (e-mail: Catherine.Potel@univ-lemans.fr).

S. Baly is now at Hautes Etudes Industrielles, 13 rue de Toul, 59046 Lille, France.

J.-F. de Belleval and P. Gatignol are with Laboratoire Roberval, UMR UTC-CNRS, Université de Technologie de Compiègne, BP 20 529, 60205 Compiegne, Cedex, France.

M. Lowe is with the Department of Mechanical Engineering, Imperial College, London SW7 2AZ, England. dress the fundamental physics and the testing applications have been published, for example in [1]-[17]. The references linked to the understanding of the present paper are more particularly detailed subsequently.

For some configurations in the ultrasonic guided wave testing of anisotropic multilayered plane structures, the waves can be locally excited in the structure by an emitter transducer immersed in an external fluid and aligned at an oblique angle to the plate. Because the incident field is a bounded beam, a Lamb wave beam is generated in the structure. If the plate material is isotropic, then the Lamb wave beam travels in the same sagittal plane as that of the transducer. However, if the material is anisotropic, then the Lamb wave beam may travel at a different angle [14], [15], [17]-[21]. Clearly it is important to understand the deviation of beam direction that may take place in order to be able to exploit these waves for testing.

The aim of this paper is to demonstrate, by means of an asymptotic approach in the far field, together with a three-dimensional (3-D) model using the decomposition of the incident beam into plane waves, and numerical and experimental results as well, how the most energetic part of the Lamb wave beam is deviated with respect to the sagittal plane of the incident bounded beam.

The useful background literature for this purpose mainly concerns the modeling of bounded beams, the interaction of such beams with a plane interface, including nonspecular reflection, and the propagation of Lamb waves.

Taking into account the geometry of the transducer leads to the concept of an ultrasonic beam. The mathematical modeling simply using monochromatic plane waves is thus no longer sufficient. Among the numerous studies of the reflection-refraction of an acoustic beam by a liquidsolid interface, the works of Ngoc and Mayer [22], then those of Pott and Harris [23], [24] can be quoted: they developed a numerical method of integration in order to calculate the intensity profile of an ultrasonic beam, especially in the neighborhood of critical Rayleigh angles, which brings out the nonspecular reflection phenomena; such behavior also was studied by Neubauer [25]. By means of a complex Laurent expansion of the reflection coefficient, Bertoni and Tamir [26], [27] developed an analytical model that describes these nonspecular phenomena. Their representation of the incident field then was used by 
Rousseau and Gatignol [28]-[30] and Matikas et al. [31], [32], who used asymptotic methods, expanded about the angles for which a Rayleigh wave propagates. They approximated the integrals of the reflected field for high frequencies, for immersed plates, and for semi-infinite media. For an arbitrary oblique incidence onto fluid/solid interfaces and on to immersed plates, the works of Bertoni and Tamir [26], [27] have been extended by Ngoc and Mayer [22] and $\mathrm{Ng}$ et al. [33]. Considering the beam as a superposition of inhomogeneous plane waves leads to similar results [34], [35].

Following some of the above-mentioned authors, the formalism used in this paper is the decomposition of the incident beam into monochromatic plane waves, and it mainly relies on works of Goodman [36], Hosten and Deschamps [37], Schaefer et al. [38] and Schaefer and Lewin [39], Souissi [40] and Belleval et al. [41], Orofino and Pedersen [42]-[48], Zeroug and Felsen [49], Rehman [50] and Rehman et al. [51].

The organization of the paper is as follows. The physical phenomenon is described by means of an asymptotic approach in Section II. This phenomenon is then numerically and experimentally illustrated in Sections III and IV: the model is first described (a 3-D model is necessary here) in Section II, numerical pressure maps are given in Section III, with a prediction of the deviation angle of the Lamb wave beam and of the oblique plane. Experimental results are given in Section IV with a comparison with numerical results.

\section{Description of the Phenomenon And Approach to Modeling}

The aim of this section is to physically describe the Lamb wave beam deviation and to explain how to predict the deviation angle, both by an asymptotic analysis and by a 3-D model using a decomposition of an ultrasonic beam into monochromatic plane waves. For background, the first part presents some results on the dispersion curves for Lamb waves in a plate, especially on the slowness curves for Lamb waves.

\section{A. Lamb Waves in an Anisotropic Multilayered Plate}

Consider an anisotropic multilayered plate immersed in a fluid. The interface plane is denoted $\left(x_{1} O x_{2}\right)$, the $x_{3}$ axis being perpendicular to the interfaces (see Fig. 1). At a characteristic pair (angle $\theta$ of the acoustic axis of the emitter transducer, frequency $f$ ), the incident acoustic beam generates locally a Lamb wave in the plate [52], [53]. The calculation of the dispersion curves for Lamb modes [54] permits the characteristic pair $(\theta, f)$ to be determined, in order to generate a Lamb wave in the plate (see for example Fig. 2 and Table I for the material characteristic of an hexagonal unidirectional composite plate [55], the fibers of which are in the direction of $x_{1}$-axis). Note that, alternatively, the phase velocity $V_{p h}$ of the Lamb wave (instead of

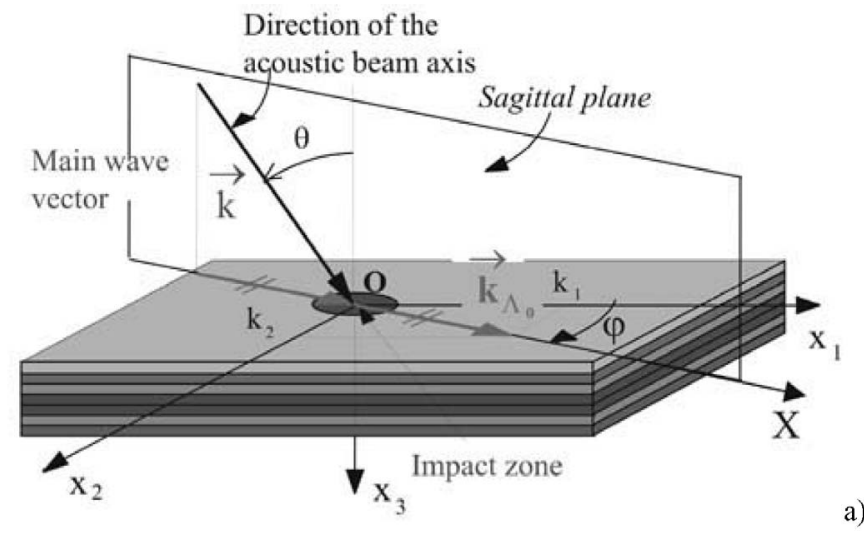

a)

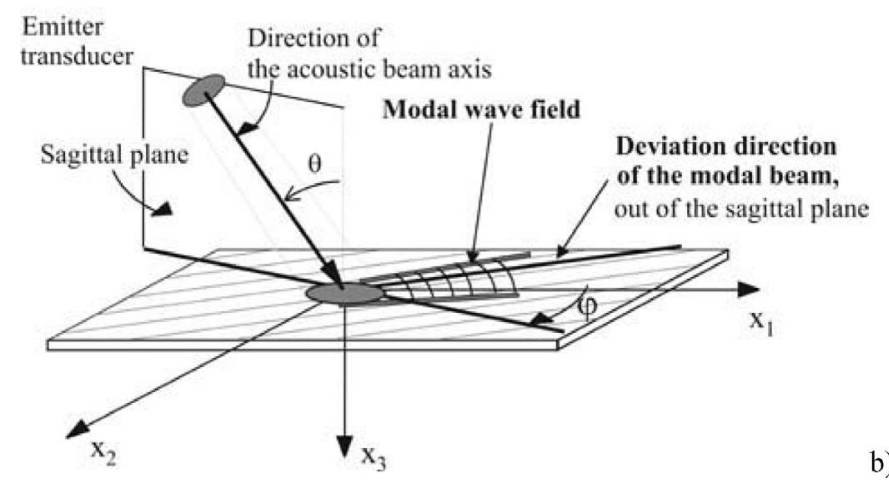

b)

Fig. 1. Geometry of the problem (a) for a multilayered anisotropic plate, (b) in the particular case of an unidirectional composite plate.

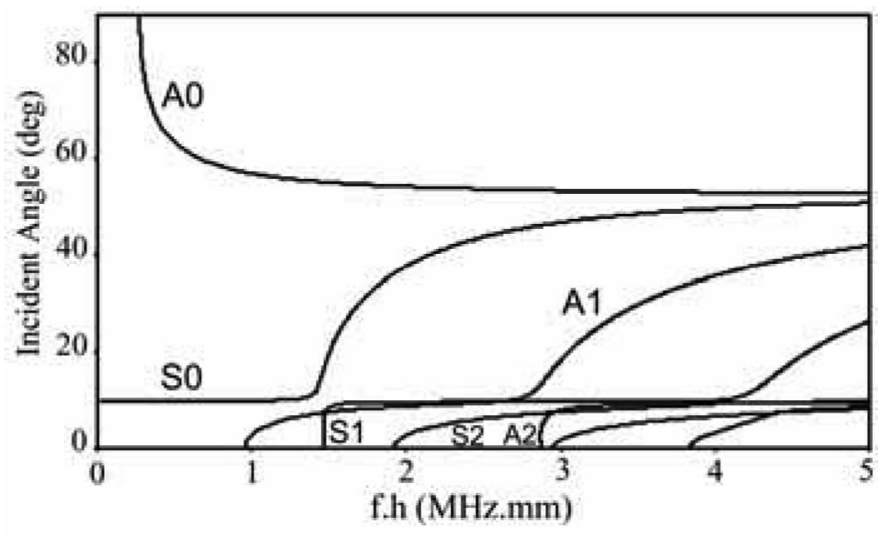

Fig. 2. Dispersion curves for Lamb waves. Unidirectional carbon/epoxy plate with sixth-order axis parallel to $x_{1}$-axis. Curves plotted using the software Disperse [53].

TABLE I

Material Characteristics of the Unidirectional Carbon-Epoxy Medium (Hexagonal Symmetry). ${ }^{1}$

\begin{tabular}{ccccccc}
\hline$C_{11}$ & $C_{12}$ & $C_{22}$ & $C_{23}$ & $C_{44}$ & $C_{55}$ & $\rho$ \\
\hline 126 & 6.7 & 13.7 & 7.1 & 3.3 & 5.8 & $1580 \mathrm{~kg} / \mathrm{m}^{3}$ \\
\hline
\end{tabular}

${ }^{1}$ Elastic constants $(\mathrm{GPa})$ such that sixth-order axis is parallel to the $x_{1}$-axis [47], with $c_{55}=\left(c_{22}-c_{23}\right) / 2$. 
the incident angle $\theta$ ) could be drawn as a function of the frequency:

$$
V_{p h}=\frac{\omega}{k_{1}}=\frac{V^{0}}{\sin \theta},
$$

where $k_{1}, \omega$, and $V^{0}$ are, respectively, the projection of the current wave number vector onto the $x_{1}$-axis, the angular frequency, and the velocity of a longitudinal wave in the fluid. These dispersion curves are obtained by writing the boundary conditions when the layer is in a vacuum, which leads to a dispersion relation of the form:

$$
\omega=F\left(k_{1}, k_{2}\right),
$$

where $k_{2}$ is the projection of the current wave number vector onto the $x_{2}$-axis.

Such curves depend on the position of the sagittal plane with respect to the plate, i.e., on the azimuthal angle $\varphi$ (see Fig. 1). The dispersion curves of Fig. 2 are drawn for a given angle $\varphi$. Actually, for realistic transducer simulation, bounded beams have to be considered, and the incident beam is centered on an acoustic axis, parallel to the main wave-vector of the beam (i.e., which conveys the maximum energy in the beam). Thus, it is necessary to represent the variation of the Lamb mode as a function of the azimuthal angle $\varphi$. When $\varphi$ varies, plotting $V_{p h}$ (or its inverse in the present case) leads to a number of modal curves $F$, given implicitly by $(2)$ in the $\left(k_{1}, k_{2}\right)$ plane. Two examples of such curves for Lamb modes are given in Fig. 3 for a carbon/epoxy layer and for two layers of a $0^{\circ} / 90^{\circ}$ carbon/epoxy structure, in the more usual form of the socalled slowness curves. Other examples may be found in [14], [18], [52]. Note that these curves are drawn for a given frequency.

\section{B. Generation of a Lamb Wave Beam by an Incident Bounded Beam-Description of the Phenomenon}

Let us consider an anisotropic, multilayered plate immersed in a fluid. In order to clarify the description of the phenomenon, the plate represented in Fig. 1(b) is an unidirectional composite plate, the fibers of which are not aligned with the direction of the $x_{1}$-axis. Note that the explanations given subsequently are very general and can be applied to the general case of a stratified medium. At a characteristic pair (angle $\theta$ of the acoustic axis of the emitter transducer, frequency $f$ ) the incident acoustic beam generates locally a Lamb wave in the structure. This Lamb beam reradiates waves into the fluid. Due to the anisotropy of the plate, the direction of the Lamb wave beam (i.e., its most energetic part) is deviated with respect to the sagittal plane (plane perpendicular to the interfaces and containing the acoustic axis of the incident beam) [19], [20]. In the case of an unidirectional plate, and according to the generated Lamb mode, it will be seen in Section III-B and Section III-C, that this deviation tends toward the direction of the fibers and can be almost parallel to them.

The above-described phenomenon, thus, has a 3-D geometry. As a consequence, a 3-D model is necessary in

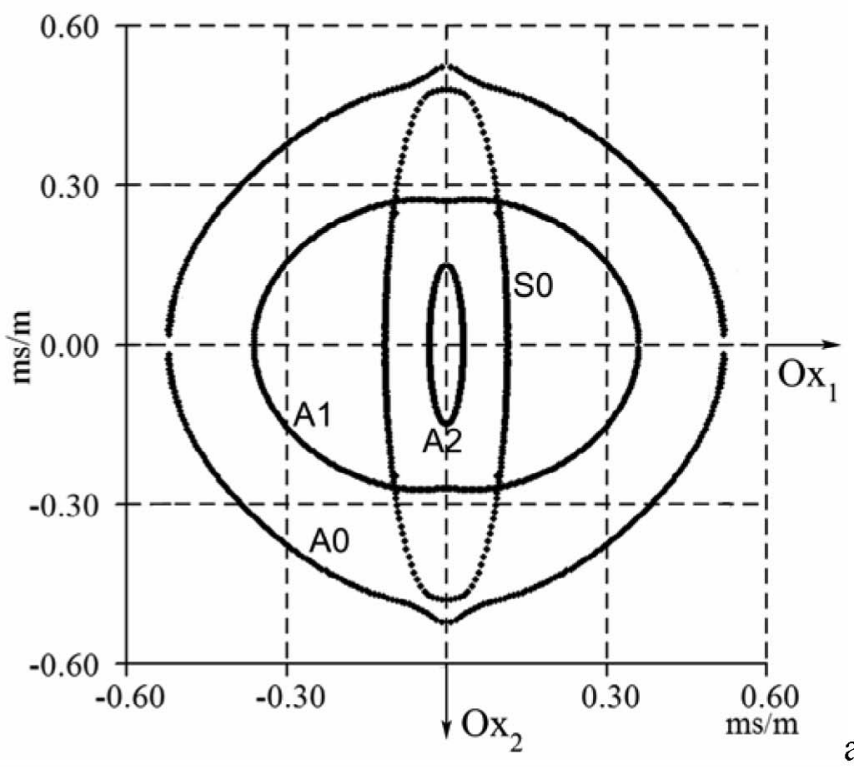

a)

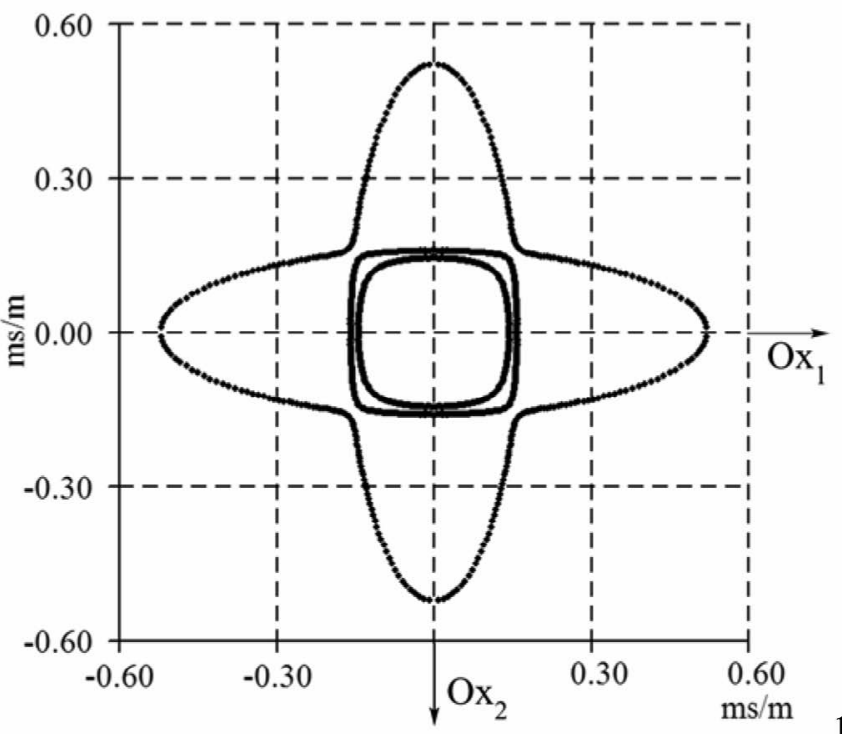

b)

Fig. 3. Slowness curves for Lamb modes f.H. $=1$ MHz.mm. (a) Unidirectional carbon/epoxy plate with sixth-order axis parallel to $x_{1}$ axis. (b) For two layers of a $0^{\circ} / 90^{\circ}$ carbon/epoxy structure.

order to simulate it and to observe the deviation of the Lamb wave beam.

\section{Description of the Model}

It is useful to recall the principle of the decomposition of a beam into monochromatic plane wave (or angular spectrum decomposition). This principle is well-known when it is applied to a scalar variable [36], [37], [41]. As mentioned in Section I, the decomposition of the incident beam into monochromatic plane waves has been used by several authors [38]-[51], and so necessarily there is some repeated information here for completeness.

Here, the formalism is applied to a vector variable, and particular attention is paid to the reference systems used (origin and basis) and to the reference of the displace- 
ment amplitude. The calculation procedure is summarized in Section III-A.

\section{Decomposition of a Beam into Monochromatic Plane Waves:}

- Consider a monochromatic plane wave with a wave number vector $\vec{k}^{E}$, propagating in a medium. A reference system $R^{E}$ is defined by a reference point $O^{E}$ and the corresponding basis $B^{E}=\left(\vec{e}_{X_{1}^{E}}, \vec{e}_{X_{2}^{E}}, \vec{e}_{X_{3}^{E}}\right)$. The representation of any point $M$ in the space is expressed as $\left(X_{1}^{E}, X_{2}^{E}, X_{3}^{E}\right)$, and the components of the vector $\vec{k}^{E}$ in the basis $B^{E}$ are denoted:

$$
\begin{array}{c|l}
\vec{k}^{E} & K_{1}^{E} \\
B^{E} & K_{2}^{E} \\
K_{3}^{E}
\end{array}
$$

The components of $\vec{k}^{E}$ are related by the following dispersion relation:

$$
\begin{aligned}
& \left(K_{1}^{E}\right)^{2}+\left(K_{2}^{E}\right)^{2}+\left(K_{3}^{E}\right)^{2}=\left(\frac{\omega}{V^{0}}\right)^{2} \\
& \text { with } k^{E}=\left\|\vec{k}^{E}\right\|=\frac{\omega}{V^{0}} .
\end{aligned}
$$

- The acoustic field, represented here by the particle displacement vector $\vec{v}^{E}$ of the considered plane wave, can be written in the following form:

$$
\begin{gathered}
\vec{v}^{E}\left(X_{1}^{E}, X_{2}^{E}, X_{3}^{E}\right)=\vec{U}^{E}\left(K_{1}^{E}, K_{2}^{E}\right) e^{-\left(i \vec{k}^{E} \cdot \overrightarrow{O^{E} M}-i \omega t\right)} \\
=\vec{U}^{E}\left(K_{1}^{E}, K_{2}^{E}\right) e^{-i\left(K_{1}^{E} X_{1}^{E}+K_{2}^{E} X_{2}^{E}+K_{3}^{E} X_{3}^{E}-\omega t\right)}
\end{gathered}
$$

where $\vec{U}^{E}\left(K_{1}^{E}, K_{2}^{E}\right)$ is referenced in $X_{3}^{E}=0$.

- Due to the linearity of the acoustic equations, a given field $\vec{u}^{E}\left(X_{1}^{E}, X_{2}^{E}, X_{3}^{E}\right)$ can be built, at any point $M$, omitting the $e^{i \omega t}$ factor, as a superposition of all the plane acoustic fields $\vec{v}^{E}\left(X_{1}^{E}, X_{2}^{E}, X_{3}^{E}\right)$, with parameters $K_{1}^{E}$ and $K_{2}^{E}$ with $K_{3}^{E}$ given by the dispersion relation (4):

$$
\begin{aligned}
& \vec{u}^{E}\left(X_{1}^{E}, X_{2}^{E}, X_{3}^{E}\right)= \\
& \frac{1}{(2 \pi)^{2}} \int_{-\infty}^{+\infty} \int_{-\infty}^{+\infty} \vec{U}^{E}\left(K_{1}^{E}, K_{2}^{E}\right) e^{-i \vec{k}^{E} \cdot \overrightarrow{O^{E} M}} d K_{1}^{E} d K_{2}^{E} .
\end{aligned}
$$

- The acoustic field $\vec{u}\left(X_{1}^{E}, X_{2}^{E}, 0\right)$ is assumed to be known in the reference plane $P_{0}^{E}=\left(O^{E}, X_{1}^{E}, X_{2}^{E}\right)$ at $X_{3}^{E}=0$. Practically, this plane is the plane of the front face of the transducer. Thus, the field $\vec{u}^{E}\left(X_{1}^{E}, X_{2}^{E}, 0\right)$ appears as the Fourier transform of the vectors $\vec{U}^{E}\left(K_{1}^{E}, K_{2}^{E}\right)$ usually called angular spectrum vectors:

$$
\begin{aligned}
\vec{u}^{E}\left(X_{1}^{E}, X_{2}^{E}, 0\right) & = \\
\frac{1}{(2 \pi)^{2}} & \int_{-\infty}^{+\infty} \int_{-\infty}^{+\infty} \vec{U}^{E}\left(K_{1}^{E}, K_{2}^{E}\right) \\
& \cdot e^{-i\left(K_{1}^{E} X_{1}^{E}+K_{2}^{E} X_{2}^{E}\right)} d K_{1}^{E} d K_{2}^{E} .
\end{aligned}
$$

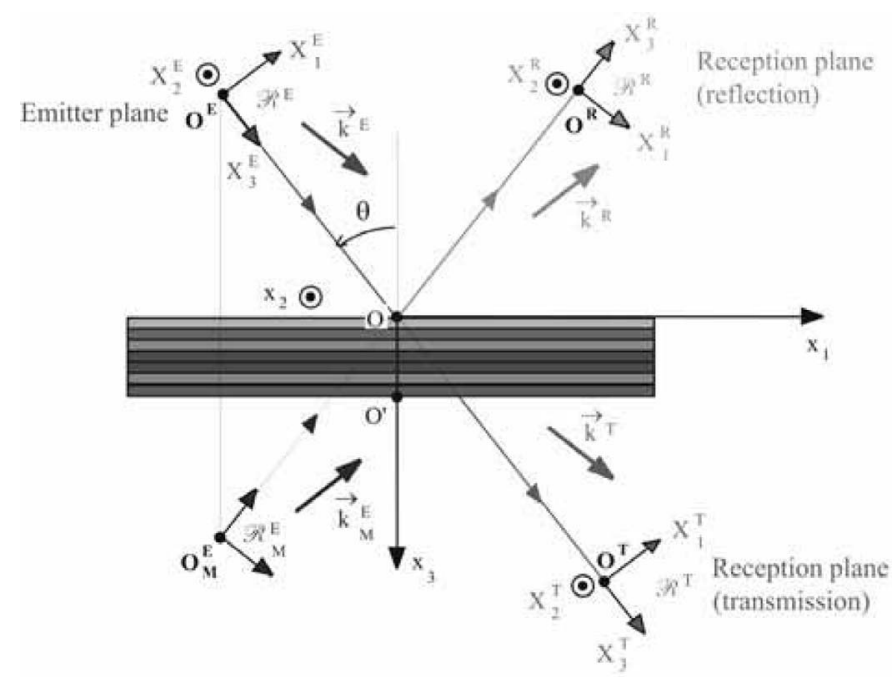

Fig. 4. Geometry of the problem for the calculation of the reflected and transmitted fields.

By an inverse Fourier transform of (7), numerically calculated by a 2-D fast Fourier transform (FFT) algorithm, the angular spectrum vector thus can be obtained in the reference plane $P_{0}^{E}$. Using (5), the angular spectrum vector in a plane $P_{Z_{0}}^{E}$, parallel to the reference plane $P_{0}^{E}$ and situated at the distance $Z_{0}$ from it, is given by:

$$
\vec{U}^{E}\left(K_{1}^{E}, K_{2}^{E} ; X_{3}^{E}=Z_{0}\right)=\vec{U}^{E}\left(K_{1}^{E}, K_{2}^{E}\right) e^{-i K_{3}^{E} Z_{0}} .
$$

The term $e^{-i K_{3}^{E} Z_{0}}$ represents a change of phase, and the particular displacement field in a plane parallel to the reference plane, is given by the Fourier transform:

$$
\begin{aligned}
& \vec{u}^{E}\left(X_{1}^{E}, X_{2}^{E}, Z_{0}\right)= \\
& \frac{1}{(2 \pi)^{2}} \int_{-\infty}^{+\infty} \int_{-\infty}^{+\infty} \vec{U}^{E}\left(K_{1}^{E}, K_{2}^{E}\right) e^{-i K_{3}^{E} Z_{0}} \\
& \cdot e^{-i\left(K_{1}^{E} X_{1}^{E}+K_{2}^{E} X_{2}^{E}\right)} d K_{1}^{E} d K_{2}^{E} .
\end{aligned}
$$

Because of the 3-D geometry, $\vec{U}^{E}\left(K_{1}^{E}, K_{2}^{E}\right)$ and $\vec{u}^{E}\left(X_{1}^{E}, X_{2}^{E}, Z_{0}\right)$ are 3 -D vectors.

This calculation is done here numerically, using a $2-\mathrm{D}$ FFT algorithm that imposes a constant step sampling. Calculating the field in a plane nonparallel to the plane $\left(O^{E}, X_{1}^{E}, X_{2}^{E}\right)$ implies a change of reference system that leads to a nonlinear relation between the former and new $K_{1}^{E}$ and $K_{2}^{E}$. As a consequence, the step of the sampling is no longer constant. It will be seen, in the next section, that the calculation of the reflected field in a plane symmetric to the emitter plane with respect to the normal to the interfaces, does not need any change in the sampling domain.

2. Interaction of the Beam with a Structure: Consider the anisotropic multilayered medium of Fig. 4 and let $R=\left(O, x_{1}, x_{2}, x_{3}\right)$ be the associated reference system. The 
emitter transducer is excited by a monochromatic signal and is immersed in a fluid. The particle displacement is assumed to be known in (or next to) the front face plane of the transducer. The normal to this plane (the acoustic axis of the transducer) makes an angle $\theta$ with the normal to the interfaces of the plate. The aim of this section is to determine the reflected field in a plane parallel to the plane symmetric to the emitter plane, with respect to the normal to the interfaces, and the transmitted field in a plane parallel to the emitter plane (see Fig. 4).

Let us define the following reference systems:

- $R^{E}=\left(O^{E}, X_{1}^{E}, X_{2}^{E}, X_{3}^{E}\right)$ with the corresponding basis $B^{E}=\left(\vec{e}_{X_{1}^{E}}, \vec{e}_{X_{2}^{E}}, \vec{e}_{X_{3}^{E}}\right)$, linked to the emitter plane,

- $R^{R}=\left(O^{R}, X_{1}^{R}, X_{2}^{R}, X_{3}^{R}\right)$ with the corresponding indirect basis $B^{R}=\left(\vec{e}_{X_{1}^{R}}, \vec{e}_{X_{2}^{R}}, \vec{e}_{X_{3}^{R}}\right)$, linked to the inspection plane for the reflected field,

- $R^{T}=\left(O^{T}, X_{1}^{T}, X_{2}^{T}, X_{3}^{T}\right)$ with the corresponding basis $B^{T}=\left(\vec{e}_{X_{1}^{T}}, \vec{e}_{X_{2}^{T}}, \vec{e}_{X_{3}^{T}}\right)=B^{E}$, linked to the inspection plane for the transmitted field.

From (6), the emitted, reflected, and transmitted displacement fields can be, respectively, written in the following form:

$$
\begin{aligned}
& \vec{u}^{E}\left(X_{1}^{E}, X_{2}^{E}, X_{3}^{E}\right)= \\
& \frac{1}{(2 \pi)^{2}} \int_{-\infty}^{+\infty} \int_{-\infty}^{+\infty} \vec{U}^{E}\left(K_{1}^{E}, K_{2}^{E}\right) e^{-i \vec{k}^{E} \cdot \overrightarrow{O^{E} M}} d K_{1}^{E} d K_{2}^{E}, \\
& \vec{u}^{R}\left(X_{1}^{R}, X_{2}^{R}, X_{3}^{R}\right)= \\
& \frac{1}{(2 \pi)^{2}} \int_{-\infty}^{+\infty} \int_{-\infty}^{+\infty} \vec{U}^{R}\left(K_{1}^{R}, K_{2}^{R}\right) e^{-i \vec{k}^{R} \cdot \overrightarrow{O^{R} M}} d K_{1}^{R} d K_{2}^{R}, \\
& \vec{u}^{T}\left(X_{1}^{T}, X_{2}^{T}, X_{3}^{T}\right)= \\
& \frac{1}{(2 \pi)^{2}} \int_{-\infty}^{+\infty} \int_{-\infty}^{+\infty} \vec{U}^{T}\left(K_{1}^{T}, K_{2}^{T}\right) e^{-i \vec{k}^{T} \cdot \overrightarrow{O^{T} M}} d K_{1}^{T} d K_{2}^{T},
\end{aligned}
$$

where $\vec{k}^{E}, \vec{k}^{R}$, and $\vec{k}^{T}$ and are the wave number vectors of respective emitted, reflected, and transmitted plane waves. Their components in the basis $B^{E}, B^{R}$, and $B^{T}$ are denoted:

$$
\vec{k}^{E} \mid \begin{array}{ll|ll|l}
K_{1}^{E} & \vec{k}^{R} & K_{1}^{R} & \vec{k}^{T} & K_{1}^{T} \\
K_{2}^{E}, & & K_{2}^{R}, & & K_{2}^{T}, \\
B_{3}^{E} & B^{R} & K_{3}^{R} & B^{R} & K_{3}^{T}
\end{array}
$$

with the representation of any point $M$ in the space in each reference system given by:

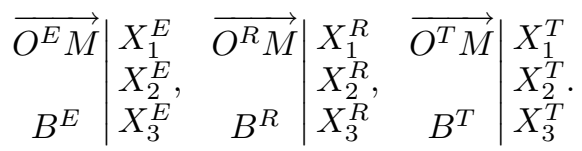

As a consequence, the displacement amplitudes of the angular spectrum vectors $\vec{U}^{E}, \vec{U}^{R}$, and $\vec{U}^{T}$ are, respectively, referenced in $O^{E}, O^{R}$, and $O^{T}$.
- For each monochromatic plane wave, the reflection and transmission coefficients $R\left(K_{1}^{E}, K_{2}^{E}\right)$ and $T\left(K_{1}^{E}, K_{2}^{E}\right)$ can be obtained by various methods in the literature, for example, by using the Thomson-Haskell method (with a change of reference of the Floquet waves) [20], [56]-[59]. Generally speaking, because of the anisotropy, the displacement fields in the plate are coupled with each other, and the displacement vectors are 3 -D vectors. The displacement amplitudes of the emitted and reflected wave are referenced at the upper interface of the structure at point $O$ (i.e., at $x_{3}=0$ ), whereas the displacement amplitude of the transmitted wave is referenced at the lower interface of the structure at point $O^{\prime}$, i.e., at $x_{3}=z_{P}$ (see Fig. 4). The phase factors are such that:

$$
\psi^{R}=\vec{k}^{E} \cdot \overrightarrow{O^{E} O}+\vec{k}^{R} \cdot \overrightarrow{O O^{R}}
$$

and

$$
\psi^{T}=\vec{k}^{E} \cdot \overrightarrow{O^{E} O}+\vec{k}^{T} \cdot \overrightarrow{O^{\prime} O^{T}} .
$$

- As the angular spectrum is known in a reference plane, the multiplication of these terms permits us to obtain the displacement fields in the reception plane, i.e., the reflected field $\vec{u}^{R}$ and the transmitted field $\vec{u}^{T}$. This multiplication is no more that a convolution in the space $\left(X_{1}^{R}, X_{2}^{R}\right)$ or $\left(X_{1}^{T}, X_{2}^{T}\right)$ :

$$
\begin{aligned}
& \vec{u}^{R}\left(X_{1}^{R}, X_{2}^{R}, X_{3}^{R}=0\right) \\
& =\frac{1}{(2 \pi)^{2}} \int_{-\infty}^{+\infty} \int_{-\infty}^{+\infty} \vec{U}_{M}^{E}\left(K_{1}^{E}, K_{2}^{E}\right) R\left(K_{1}^{E}, K_{2}^{E}\right), \\
& \cdot e^{-i \psi^{R}} e^{-i\left(K_{1}^{R} X_{1}^{R}+K_{2}^{R} X_{2}^{R}\right)} d K_{1}^{R} d K_{2}^{R}
\end{aligned}
$$

and

$$
\begin{aligned}
& \vec{u}^{T}\left(X_{1}^{T}, X_{2}^{T}, X_{3}^{T}=0\right) \\
& =\frac{1}{(2 \pi)^{2}} \int_{-\infty}^{+\infty} \int_{-\infty}^{+\infty} \vec{U}^{E}\left(K_{1}^{E}, K_{2}^{E}\right) T\left(K_{1}^{E}, K_{2}^{E}\right), \\
& \cdot e^{-i \psi^{T}} e^{-i\left(K_{1}^{T} X_{1}^{T}+K_{2}^{T} X_{2}^{T}\right)} d K_{1}^{T} d K_{2}^{T}
\end{aligned}
$$

where $\vec{U}_{M}^{E}\left(K_{1}^{E}, K_{2}^{E}\right)$ is the angular spectrum vector mirror to the incident angular spectrum vector $\vec{U}^{E}\left(K_{1}^{E}, K_{2}^{E}\right)$ with respect to the interfaces (see Fig. 4). Due to the choice of the basis $B^{E}, B^{R}$, and $B^{R}$, the components of the incident, reflected and transmitted wave number vectors in these bases are equal. Let us note:

$$
K_{1}=K_{1}^{E}=K_{1}^{R}=K_{1}^{T} \text { and } K_{2}=K_{2}^{E}=K_{2}^{R}=K_{2}^{T} .
$$

As a consequence, the reflected angular spectrum vector $\vec{U}^{R}\left(K_{1}, K_{2}\right)$ is equal, omitting the factor $R\left(K_{1}, K_{2}\right) \cdot e^{-i \psi^{R}}$, to the mirror angular spectrum vector $\vec{U}_{M}^{E}\left(K_{1}, K_{2}\right)$. The components of $\vec{U}_{M}^{E}\left(K_{1}, K_{2}\right)$ in the 
basis $B^{R}$ are the same as the components of $\vec{U}^{E}\left(K_{1}, K_{2}\right)$ in the basis $B^{E}$, i.e., if:

$$
\vec{U}^{E}\left(K_{1}^{E}, K_{2}^{E}\right)=\sum_{i=1}^{3} U_{i}^{E}\left(K_{1}^{E}, K_{2}^{E}\right) \vec{e}_{X_{i}^{E}}
$$

then

$$
\vec{U}_{M}^{E}\left(K_{1}^{E}, K_{2}^{E}\right)=\sum_{i=1}^{3} U_{i}^{E}\left(K_{1}^{E}, K_{2}^{E}\right) \vec{e}_{X_{i}^{R}} .
$$

And:

$$
\begin{aligned}
& \vec{u}^{R}\left(X_{1}^{R}, X_{2}^{R}, X_{3}^{R}=0\right) \\
& =\frac{1}{(2 \pi)^{2}} \int_{-\infty}^{+\infty} \int_{-\infty}^{+\infty} \vec{U}_{M}^{E}\left(K_{1}, K_{2}\right) R\left(K_{1}, K_{2}\right) \\
& \cdot e^{-i \psi^{R}} e^{-i\left(K_{1} X_{1}^{R}+K_{2} X_{2}^{R}\right)} d K_{1} d K_{2},
\end{aligned}
$$

and

$$
\begin{aligned}
& \vec{u}^{T}\left(X_{1}^{T}, X_{2}^{T}, X_{3}^{T}=0\right) \\
& =\frac{1}{(2 \pi)^{2}} \int_{-\infty}^{+\infty} \int_{-\infty}^{+\infty} \vec{U}^{E}\left(K_{1}, K_{2}\right) T\left(K_{1}, K_{2}\right) \\
& \cdot e^{-i \psi^{T}} e^{-i\left(K_{1} X_{1}^{T}+K_{2} X_{2}^{T}\right)} d K_{1} d K_{2} .
\end{aligned}
$$

Knowing that, generally speaking, the pressure is related to the $X_{3}$-component of the particle displacement by the following formula:

$$
P\left(K_{1}, K_{2} ; \omega\right)=\frac{i \rho^{0} \omega^{2}}{K_{3}} U_{3}\left(K_{1}, K_{2} ; \omega\right),
$$

where $\rho^{0}$ is the density of the fluid, the reflected and transmitted pressure fields are, respectively, given by:

$$
\begin{aligned}
& P^{R}\left(X_{1}^{R}, X_{2}^{R}, X_{3}^{R}=0\right) \\
& =\frac{i \rho^{0} \omega^{2}}{(2 \pi)^{2}} \int_{-\infty}^{+\infty} \int_{-\infty}^{+\infty} \frac{1}{K_{3}^{R}} U_{M_{3}}^{E}\left(K_{1}, K_{2}\right) R\left(K_{1}, K_{2}\right), \\
& \cdot e^{-i \psi^{R}} e^{-i\left(K_{1} X_{1}^{R}+K_{2} X_{2}^{R}\right)} d K_{1} d K_{2}
\end{aligned}
$$

and

$$
\begin{aligned}
& P^{T}\left(X_{1}^{T}, X_{2}^{T}, X_{3}^{T}=0\right) \\
& =\frac{i \rho^{0} \omega^{2}}{(2 \pi)^{2}} \int_{-\infty}^{+\infty} \int_{-\infty}^{+\infty} \frac{1}{K_{3}^{T}} U_{3}^{E}\left(K_{1}, K_{2}\right) T\left(K_{1}, K_{2}\right), \\
& \cdot e^{-i \psi^{T}} e^{-i\left(K_{1} X_{1}^{T}+K_{2} X_{2}^{T}\right)} d K_{1} d K_{2}
\end{aligned}
$$

where $U_{M_{3}}^{E}=U_{3}^{E}$ (20b). Due to (19), the sampling is the same in the emitted, reflected, and transmitted planes.

It should be noted that the calculation of the reflected and transmitted fields in other planes, in particular in a plane parallel to the interfaces of the plate, would require a Jacobian term in the integrals. The numerical procedure in order to avoid it is described in Section III-A.

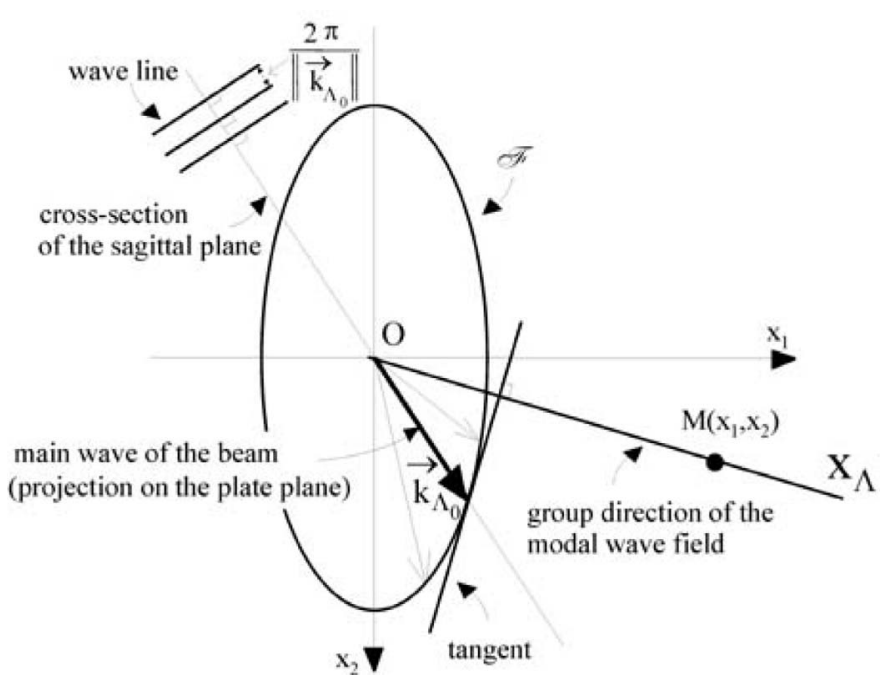

Fig. 5. Obtaining the main group direction of the modal wave field, for a particular branch of the modal curve.

\section{Asymptotic Analysis by a Stationary Phase Argument}

The above-described model permits one to numerically obtain reflected and transmitted fields of pressure in the fluid surrounding the immersed plate, especially when, at a characteristic pair (angle $\theta$ of the acoustic axis of the emitter transducer, frequency $\omega$ ), the incident acoustic beam generates locally a Lamb wave in the plate. The deviation phenomenon, described above, thus can be numerically illustrated, and the deviation angle of the Lamb beam can be numerically found (see below). However, an asymptotic analysis in the far field also permits one to predict this deviation angle, using the Lamb slowness curves. The aim of this section is to summarize the reasoning for this asymptotic analysis; full details are contained in [18]-[20].

Consider the point corresponding to the projection $\vec{k}_{\Lambda_{0}}$ of the main wave vector of the acoustic axis of the transducer onto the plate (see Fig. 1) on one branch of the slowness Lamb curve (see Fig. 5). The incident beam also generates waves with slowness vectors close to this point of the curve. These waves contribute to the modal propagation and, therefore, a bounded Lamb beam is generated in the structure. By means of a stationary phase argument, it is possible to demonstrate that the most energetic part of this modal beam propagates along the group direction. This group direction is along the normal to the Lamb slowness curve at the point on the curve given by the direction of the Lamb wave vector. As the medium constituting the layers is anisotropic, the Lamb slowness curve is not circular and this normal direction is different from the direction of the Lamb wave vector $\vec{k}_{\Lambda_{0}}$. As a consequence, the Lamb wave beam is deviated in the group direction $x_{\Lambda}$ associated with the modal wave vector of the acoustic axis of the incident beam (see Fig. 5). It should be noted here that, as the phenomenon is described for a monochromatic field, the only dispersion that is involved in the problem is the angular dispersion of the Lamb waves. Thus, it is the group 
a)
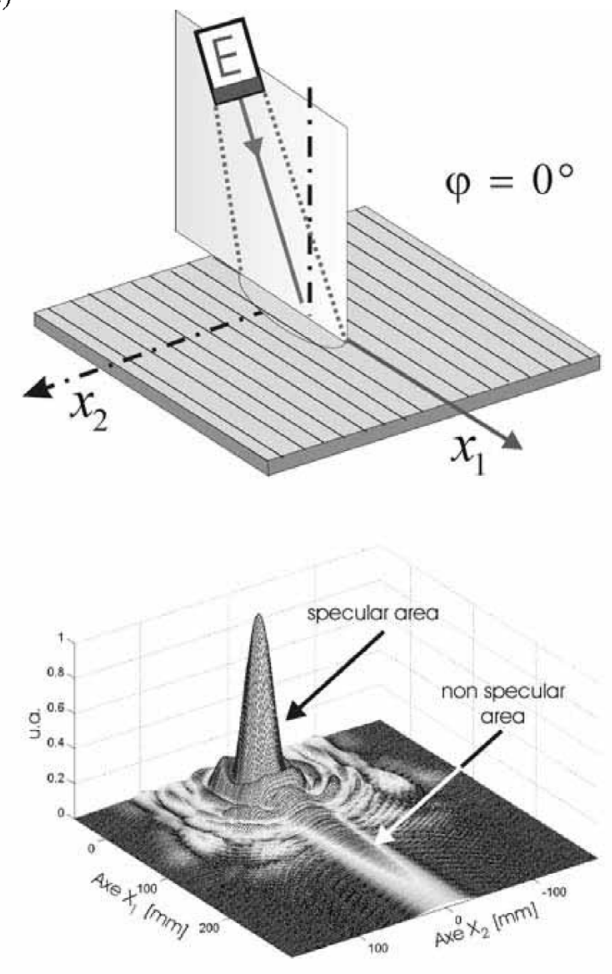

b)
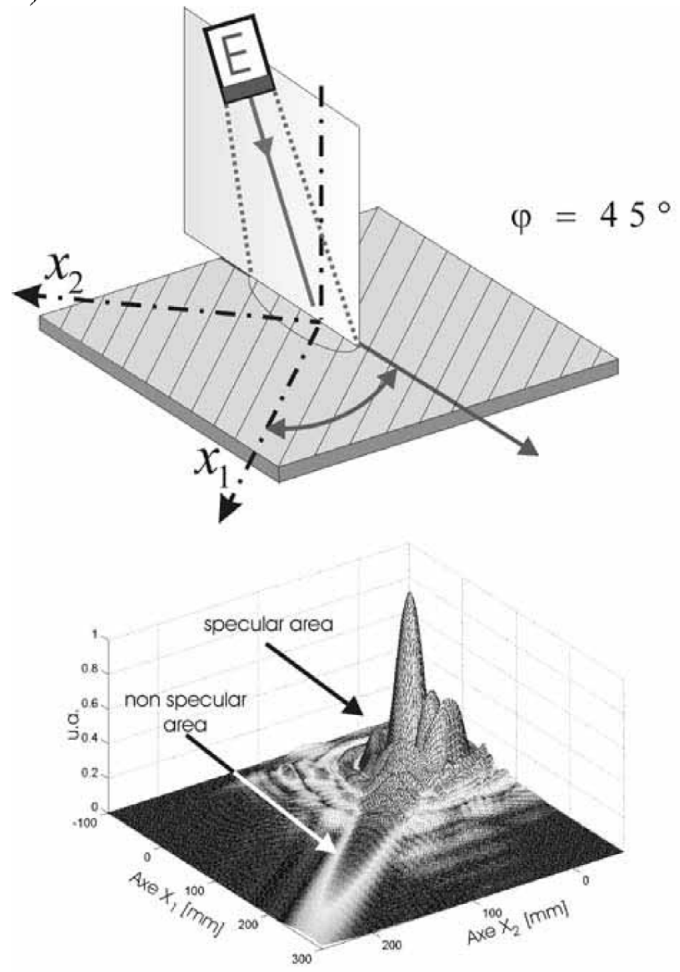

Fig. 6. Reflected field of pressure, in a plane parallel to a unidirectional carbon/epoxy plate with thickness $H=0.59 \mathrm{~mm}, f=1.35 \mathrm{MHz}$, mode $S_{0}$. (a) Sixth-order axis parallel to $x_{1}$-axis, $\varphi=0^{\circ}$. (b) Sixth-order axis not parallel to $x_{1}$-axis, $\varphi=45^{\circ}$.

direction that is important in this case, and not the group velocity.

The modal beam, as it propagates along the anisotropic medium, radiates into the external fluid. This radiation phenomenon is centered in an oblique plane corresponding to the reflected direction of the acoustic axis. The intersection of this oblique plane with the interface direction is the main group direction of the modal beam. All the nonspecular effects observed by Neubauer [25] and modeled by several authors, now have to be searched in this oblique plane, and not in the sagittal plane. Thus in this context, because of the beam deviation, the usual 2-D modeling of the acoustic beam is no longer sufficient, and the 3-D model is needed.

\section{Numerical Method And Results}

Using the numerical procedure presented in Section III, corresponding to the model described in Section II, numerical results are presented for carbon/epoxy structures, in order to illustrate the deviation of the Lamb wave beam. A first illustration of the phenomenon is given in Section III, with the conventions of representation used here, then several maps of the reflected field are given in Section III, with a comparison between the deviation angle given numerically by the model and by the asymptotic analysis explained in Section II. The radiation of the reflected field in an oblique plane also is illustrated.

\section{A. Calculation Procedure}

The calculation procedure corresponding to the 3-D model described in Section II is as follows:

- The particle displacement is simulated in the emitter transducer plane by an analytical expression. Experimental measurements (in order to take into account a real transducer) also could be used.

- The incident field is decomposed into plane waves, using a 2-D FFT algorithm.

- For each oblique monochromatic plane wave, reflection and transmission coefficients are calculated using the transfer matrix formalism. Alternative multilayered models from the literature also could be used here.

- A Fourier transform, numerically calculated by a $2-\mathrm{D}$ IFFT algorithm, permits us to obtain the displacement and pressure fields in the spatial domain.

- According to the applications (propagation of Lamb waves for example), an interpolation in the spatial domain permits us to obtain the transmitted and reflected fields of pressure in a plane parallel to the interfaces of the plate.

Note that the reflected field is numerically calculated in a plane parallel to the plane that is symmetric to the emitter plane, with respect to the normal to the interfaces; and the transmitted field is calculated in a plane parallel to the emitter plane. The calculation of these fields in a plane parallel to the interfaces of the plate would require 


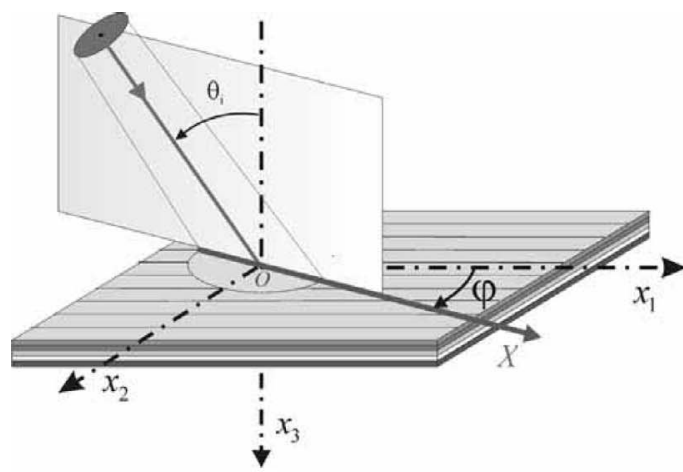

a)

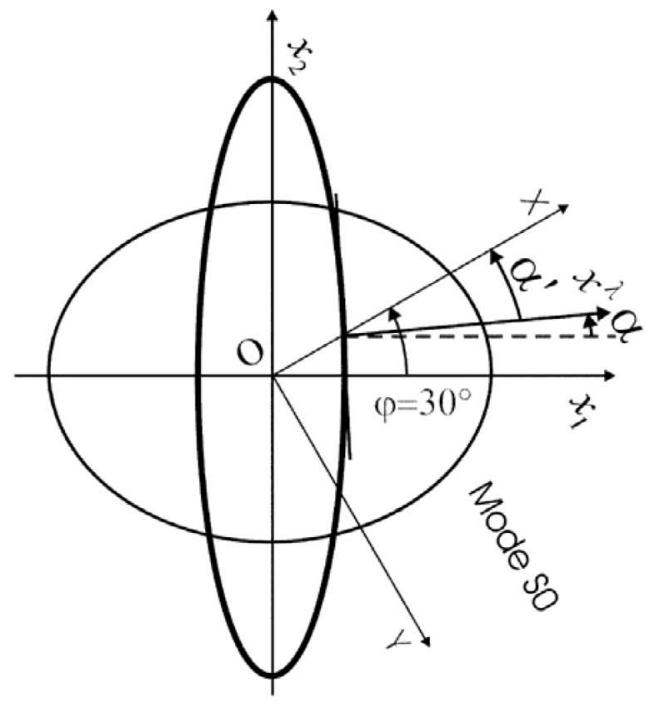

b)

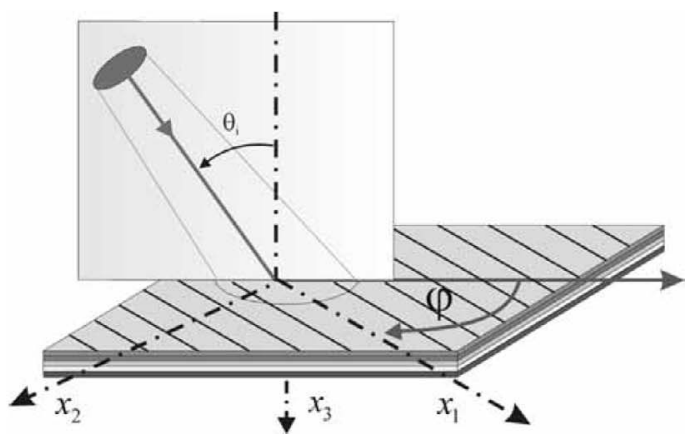

c)

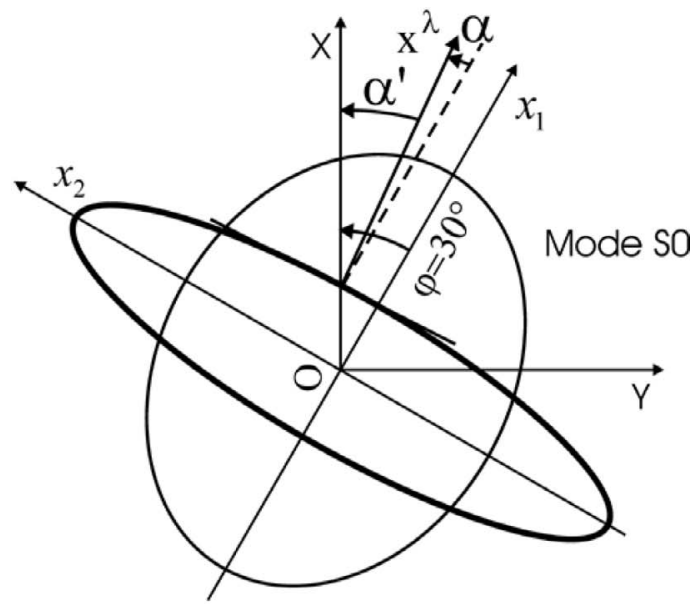

d)

Fig. 7. Two points of view for the representation of the results: (a) and (b) first point of view, rotation of the sagittal plane; (c) and (d) second point of view, rotation of the plate; (b) and (d) slowness curves.

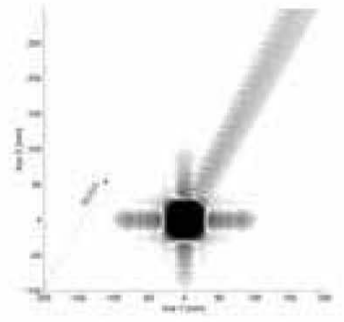

a)

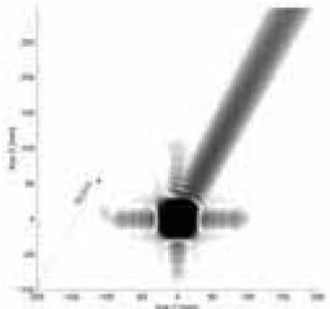

$3=-1+\cdots$ b)

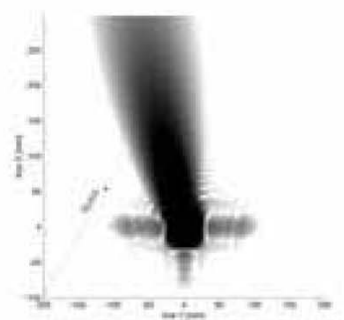

c)

Fig. 8. Map of the reflected field of pressure for a unidirectional carbon/epoxy plate $(f . H .=1 \mathrm{MHz} \cdot \mathrm{mm})$, azimuthal angle $\varphi=30^{\circ}$. Excitation of (a) mode $S_{0} \theta=11.2^{\circ}$, (b) mode $A_{2} \theta=3.1^{\circ}$, (c) mode $A_{1} \theta=29.5^{\circ}$.

both the calculation of a Jacobian in the integrals, and an interpolation of the sampling. This choice of planes thus avoids that additional complication. Then, in order to find the field of pressure in a plane parallel to the interfaces of the plate, the pressure is calculated for a set of planes of the reflected or transmitted field, each one with a different distance along the reflected or transmitted beam path. The required field then is found from the intersection of the set of planes with the plane parallel to the interfaces of the plate.
This model is very general because it takes into account the anisotropy of each layer constituting the structure, the geometry of the transducer, and the propagation in three dimensions.

\section{B. First Illustration of the Lamb Wave Beam Deviation}

In this illustration, the reflected fields of pressure are represented in a plane parallel to the plate. The position of the sagittal plane is located by the azimuthal angle $\varphi$ (see 


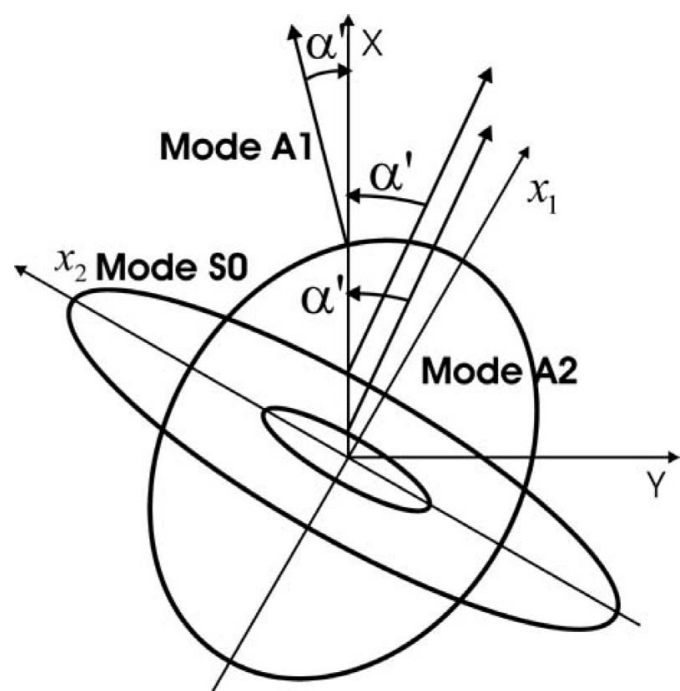

Fig. 9. Slowness curves for Lamb modes for a unidirectional carbon/epoxy plate $(f . H .=1 \mathrm{MHz} . \mathrm{mm})$ with the deviation angle $\alpha^{\prime}$ associated to each mode, when $\varphi=30^{\circ}$.

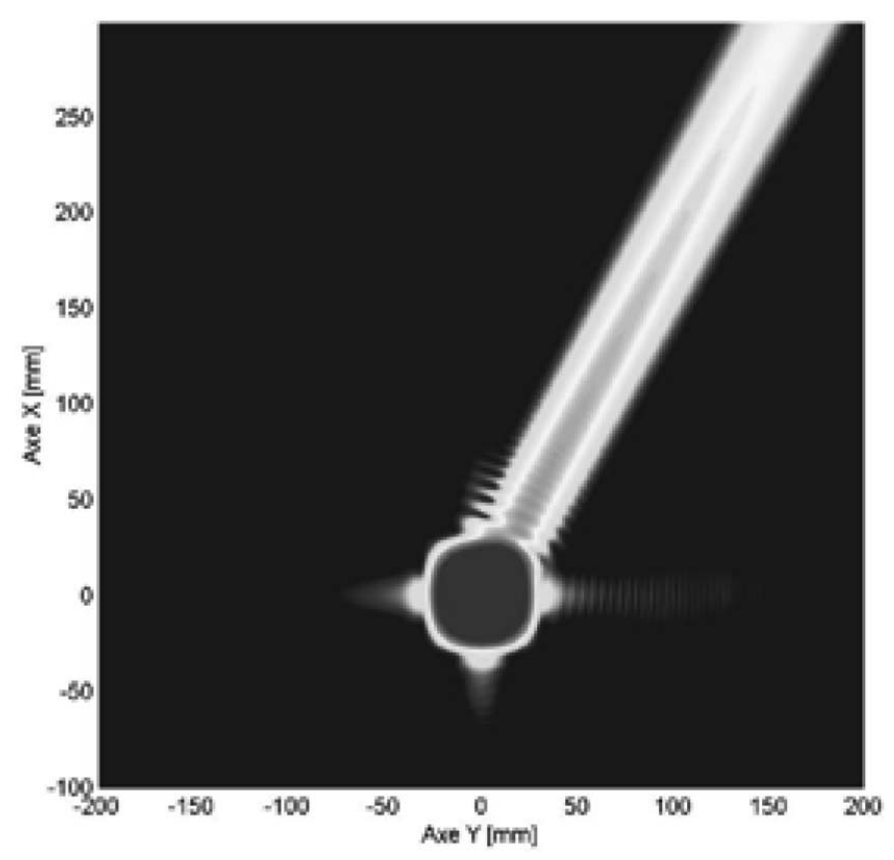

Fig. 10. Influence of the number of points for defining numerically the emitter transducer (20 points).

Fig. 1). Let $O X$ be the intersection of the sagittal plane with the first interface of the plane [see Figs. 1 and 6(a)]. Thus, all the reflected fields of pressure are represented as maps in the plane $(O X, O Y)$, the $O Y$-axis being perpendicular to the $O X$-axis. This simply amounts to making the plate rotate while keeping the sagittal plane fixed, instead of making the sagittal plane vary while keeping the plate fixed. These two points of view are quite equivalent (apart from the signs of angles).

A first illustration of the Lamb wave beam deviation is presented in Fig. 6, for a unidirectional carbon/epoxy plate, when the fibers' direction is contained in the sagit-

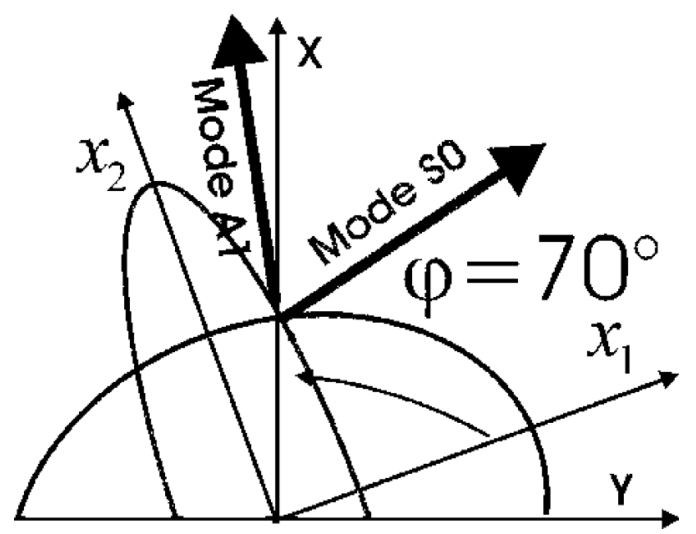

Fig. 11. Crossing of the Lamb slowness curves of a unidirectional carbon/epoxy plate $(f . H .=1 \mathrm{MHz} . \mathrm{mm})$ for modes $S_{0}$ and $A_{1}, \varphi=$ $70^{\circ}$.

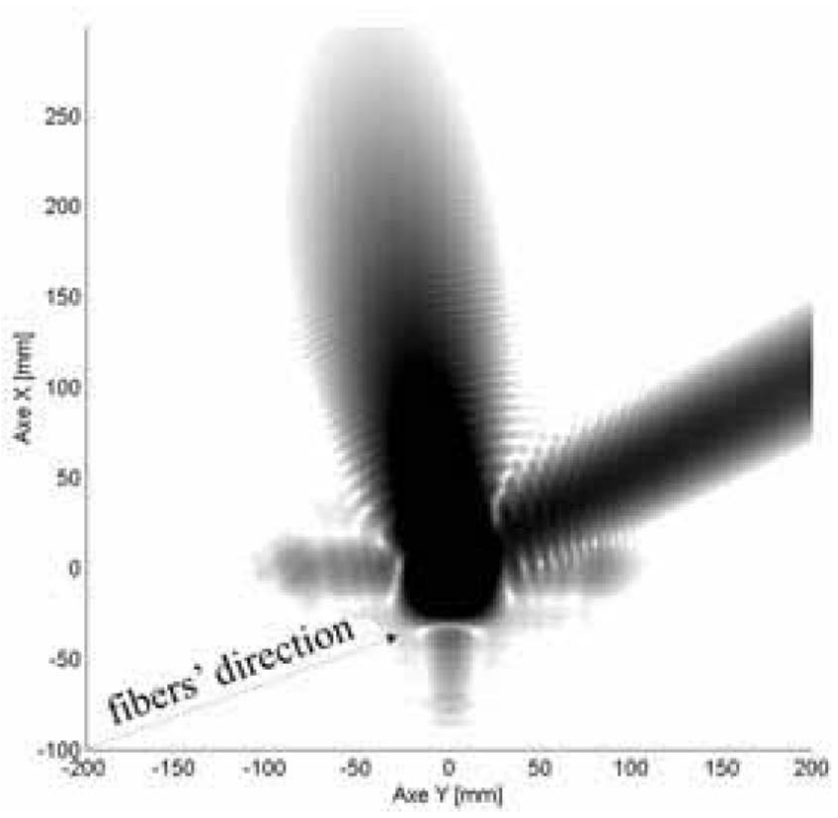

Fig. 12. Map of the reflected field of pressure for a unidirectional carbon/epoxy plate $(f . H .=1 \mathrm{MHz} . \mathrm{mm})$, azimuthal angle $\varphi=70^{\circ}$. Excitation of modes $S_{0}$ and $A_{1}$.

tal plane [see Fig. 6(a)]; in this case, as expected, the most energetic part of the nonspecular field is not deviated with respect to the sagittal plane. However, when the fibers' direction is not contained in the sagittal plane [see Fig. 6(b)], a deviation of the Lamb wave field is observed. Here, in this particular case, the deviation direction is practically to that of the fibers. In both cases, the specular and nonspecular parts of the reflected field can be observed.

\section{Study of Several Lamb Modes}

It has been seen that, in the far field (corresponding to the nonspecular part of the reflected field), the Lamb wave beam generated in the structure is centered on a direction $x_{\Lambda}$ given by the normal to the slowness curve at the point corresponding to the acoustic axis of the emitter. Let us 


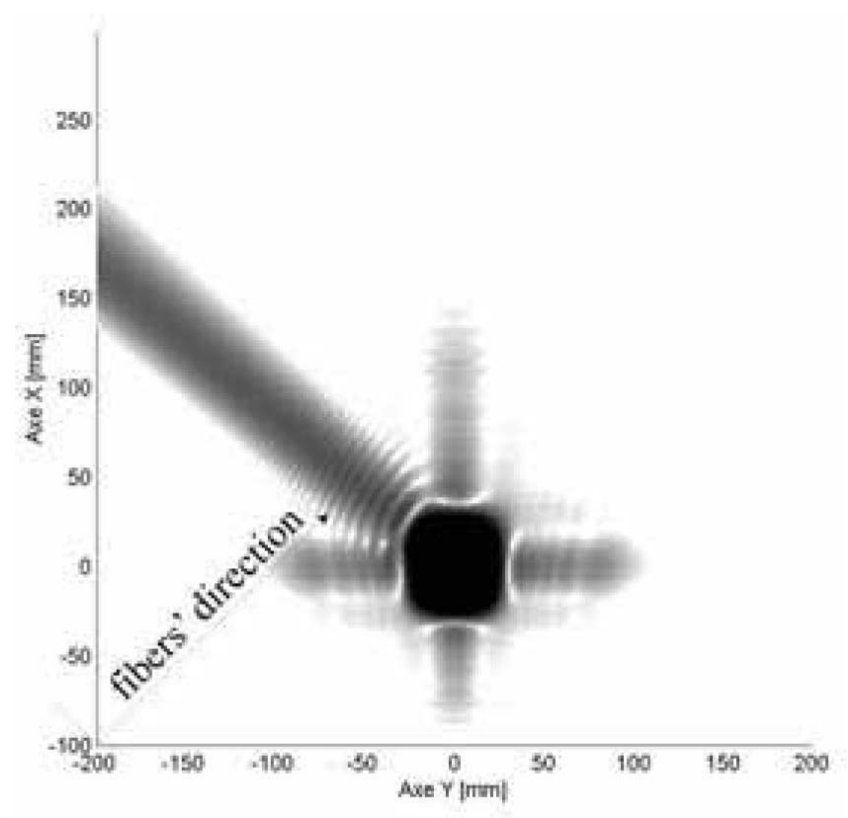

Fig. 13. Map of the reflected field of pressure for two layers of a $0^{\circ} / 90^{\circ}$ carbon/epoxy structure $(f . H .=1 \mathrm{MHz} \cdot \mathrm{mm})$, azimuthal angle $\varphi=30^{\circ}$, incident angle $\theta=24.7^{\circ}$. Excitation of mode 3 [see Fig. 3(b)].

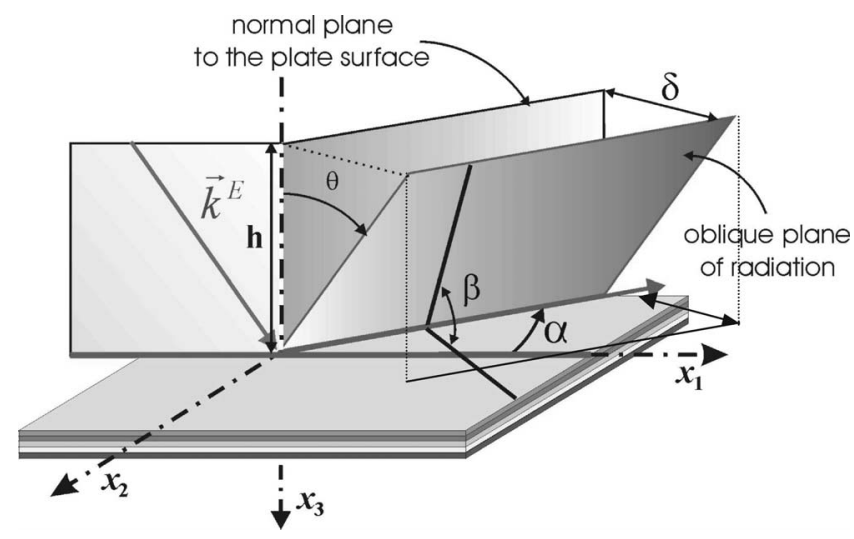

Fig. 14. Radiation of the Lamb beam field in an oblique plane in the external fluid.

note $\alpha^{\prime}$ the angle between the $x_{\Lambda}$-axis and the $O X$ axis, and let us call it the deviation angle.

It also is possible to determine the group direction from the numerical results of the above-described model by seeking the maximum amplitude of the reflected field of pressure. Considering only the nonspecular part of the reflected field, it, thus, is possible to determine the deviation angle of the Lamb wave beam.

The following maps are presented together with the associated Lamb slowness curves and their corresponding group directions (normal to the slowness curve). Figs. 7(b) and (d) present the Lamb slowness curves for an unidirectional carbon/epoxy plate (modes $S_{0}$ and $A_{1}$ ), with the fiber direction parallel to the $x_{1}$-axis, with the two points of view for the representation of the maps. All the maps are presented using the second point of view of Fig. $7(\mathrm{~d})$,
TABLE II

Lamb Mode Deviation Obtained by Both Theoretical and Numerical Methods. ${ }^{1}$

\begin{tabular}{lccc}
\hline Lamb mode & $A_{2}$ & $S_{0}$ & $A_{1}$ \\
\hline Normal to slowness curve for Lamb & $28.0^{\circ}$ & $28.0^{\circ}$ & $-12.0^{\circ}$ \\
Maximum of magnitude of pressure & $27.9^{\circ}$ & $28.3^{\circ}$ & $-10.6^{\circ}$ \\
\hline
\end{tabular}

${ }^{1}$ Uniaxial carbon-epoxy plate, azimuthal angle $\varphi=30^{\circ}$, frequencythickness $=1$ MHz.mm.

which is a simple rotation of the azimuthal angle $\varphi$ of Fig. 7(b). The distance between the emitter and the plate is equal to $200 \mathrm{~mm}$. The emitter has a Gaussian response, with a diameter equal to $20 \mathrm{~mm}$. The reflected pressure is calculated at the surface of the plate, in water.

Fig. 8 presents the map of the reflected field of pressure for an unidirectional carbon/epoxy plate, when the fiber direction is not contained in the sagittal plane (azimuthal angle $\varphi=30^{\circ}$ ). The associated slowness curves are given in Fig. 9. The excited Lamb mode is not the same for Figs. 8(a), (b), and (c). It appears clear that the deviation direction of the Lamb mode depends on the excited mode itself. Indeed, these three excited modes are those described in Fig. 9. From Fig. 9, it can be observed that modes $A_{2}$ and $S_{0}$ have slowness curves with very similar shapes, whereas that of mode $A_{1}$ is totally different. As a result, the direction of propagation of Lamb mode $A_{1}$ is different from that of modes $A_{2}$ and $S_{0}$, which roughly follow the fiber direction. The angles of deviation for each mode are given in Table II, using the normal to the slowness curves (asymptotic approach method) and the search for the maximum amplitude of the reflected field of pressure (numerical method). It can be seen that both methods are in excellent agreement. The amplitude of the reflected field depends on the coupling effect between the fluid and the structure.

Note that the cross shape located at the specular reflected field, which is visible on all maps, is related to the number of points taken to define the emitter. Here, for computational time reasons, this number of points is equal to 10 . For more points, the cross is less visible, as can be seen, for example, in Fig. 10.

When two Lamb slowness curves cross $\left(\varphi=70^{\circ}\right.$, see Fig. 11), both modes $A_{1}$ and $S_{0}$ are excited, as can be seen in Fig. 12. As the shape of these two slowness curves is very different, so are the normal and thus the deviation angles. It is important to recognize that such a phenomenon can be observed only because of the $3-\mathrm{D}$ model and because the spatially finite nature of the ultrasonic beam is taken into account.

Let us now consider a structure made up of two carbon/epoxy layers, the fibers of one being perpendicular to those of the other $\left(0^{\circ} / 90^{\circ}\right.$ structure $)$, each layer being 0.11-mm thick. The corresponding Lamb slowness curves are given in Fig. 3(b) and the predicted map is shown in Fig. 13. The deviation angle given by the normal to the slowness curve $\left(\alpha^{\prime}=-50^{\circ}\right)$ is in very good agreement 


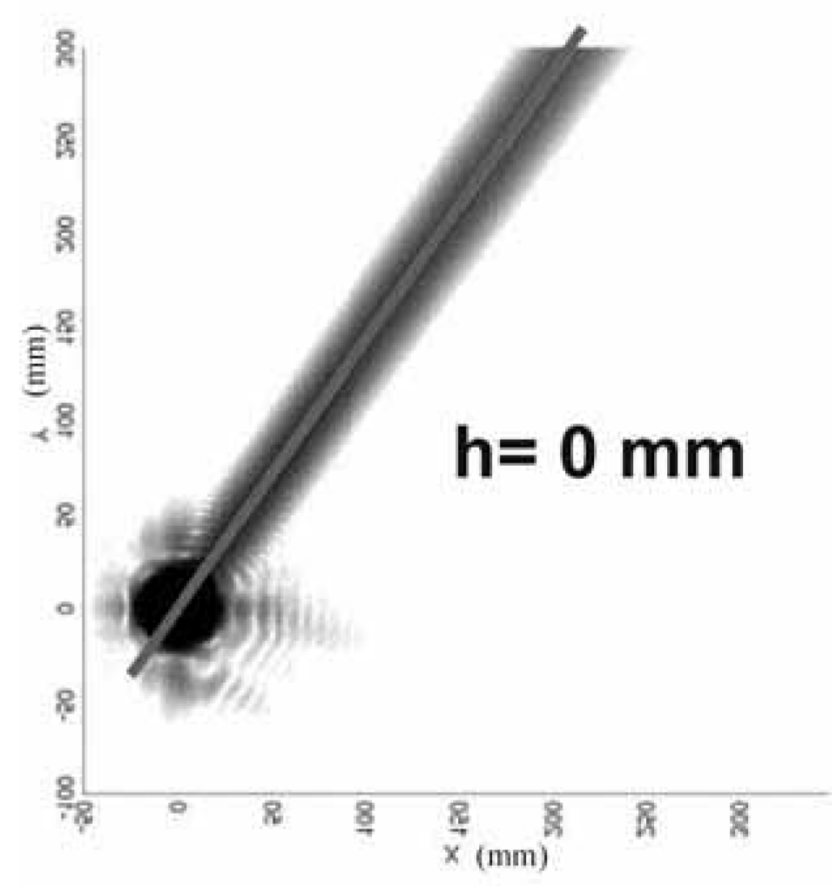

a)

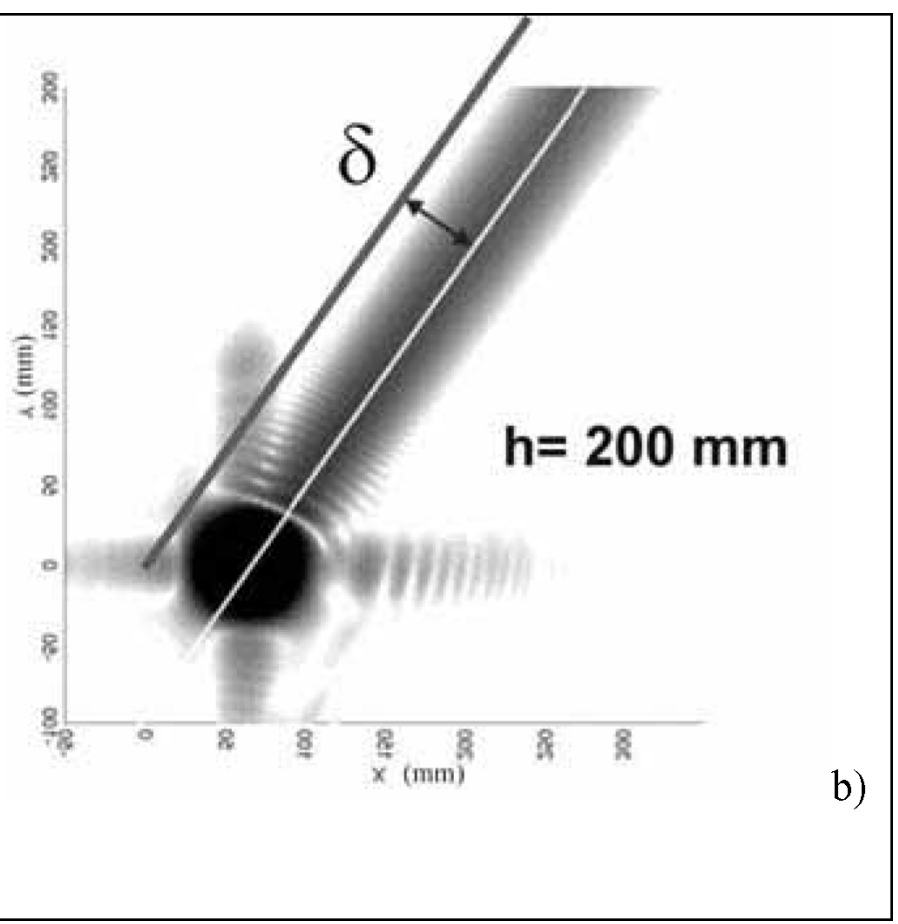

Fig. 15. Maps of the reflected field of pressure for a unidirectional carbon/epoxy plate $(f \cdot H .=1 \mathrm{MHz} \cdot \mathrm{mm})$, azimuthal angle $\varphi=60^{\circ}$. Excitation of mode $S_{0}$. (a) $h=0 \mathrm{~mm}$, (b) $h=200 \mathrm{~mm}$.

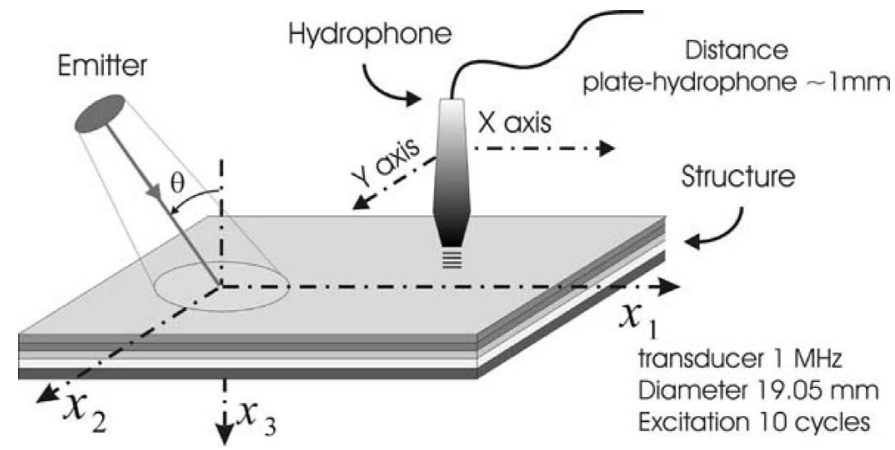

Fig. 16. Experimental setup.

with that given by the maximum of the magnitude of the pressure $\left(\alpha^{\prime}=-50 \cdot 1^{\circ}\right)$.

\section{Radiation in an Oblique Plane}

The Lamb wave beam reradiates into the external fluid in an oblique plane corresponding to the specular reflection angle of the acoustic axis (see Fig. 14). The field in this oblique plane has been studied by an asymptotic analysis in [19], [20]. In this section, it is illustrated numerically.

Let $\beta$ be the angle between the oblique plane and the plate, and $\delta$ be the distance between this plane and the $O x_{3}$-axis, for a given height $h$ (see Fig. 14). If the reflected field is examined at the height $h$ from the plate, the maximum of the magnitude of the pressure is found in the oblique plane, and its projection onto the plane of the plate is located at the distance $\delta$ from the $O x_{\Lambda}$-axis (main group direction). Knowing the incident angle $\theta$ and the deviation angle $\alpha=\left(O x_{1}, O x_{\Lambda}\right)$, a simple geometric calculation leads to the following formulae $\left(\varphi=0^{\circ}\right)$ :

$$
\beta=\operatorname{Arctg}\left(\frac{1}{\operatorname{tg} \theta \sin \alpha}\right)=\frac{\pi}{2}-\operatorname{Arctg} \frac{\delta}{h} .
$$

Numerically, Fig. 15(b) presents the reflected field of pressure for a unidirectional carbon/epoxy plate when $h=200 \mathrm{~mm}$. The dotted line corresponds to the maximum magnitude of pressure when $h=0$, transferred from Fig. 15(a). It can be observed clearly that the two Lamb wave beams are shifted. The measurement of the distance $\delta$ permits us to determine the angle $\beta$, using (24). The theoretical value coming from $(24)\left(\beta=74.7^{\circ}\right)$ and the numerical value $\left(\beta=75.3^{\circ}\right)$ are in very good agreement. It also can be seen that there is an increase of the Lamb wave beam size as a function of the height $h$, which is due to the spreading of the beam.

\section{EXPERIMENTAL RESUlts}

Experiments have been made on a $0.59-\mathrm{mm}$ thick unidirectional carbon/epoxy plate in order to validate the developments.

\section{A. Experimental Setup}

The emitting transducer used to generate the ultrasonic field is a Panametrics wide band (Panametrics-NDT) PZT device, V314, with nominal frequency $1 \mathrm{MHz}$ and diameter $3 / 4$ inch $(19.05 \mathrm{~mm})$. The receiver is a needle hydrophone, PVDF technology, diameter $1 \mathrm{~mm}$, from Precision Acoustics Ltd., Dorchester, Dorset, England. The hydrophone 

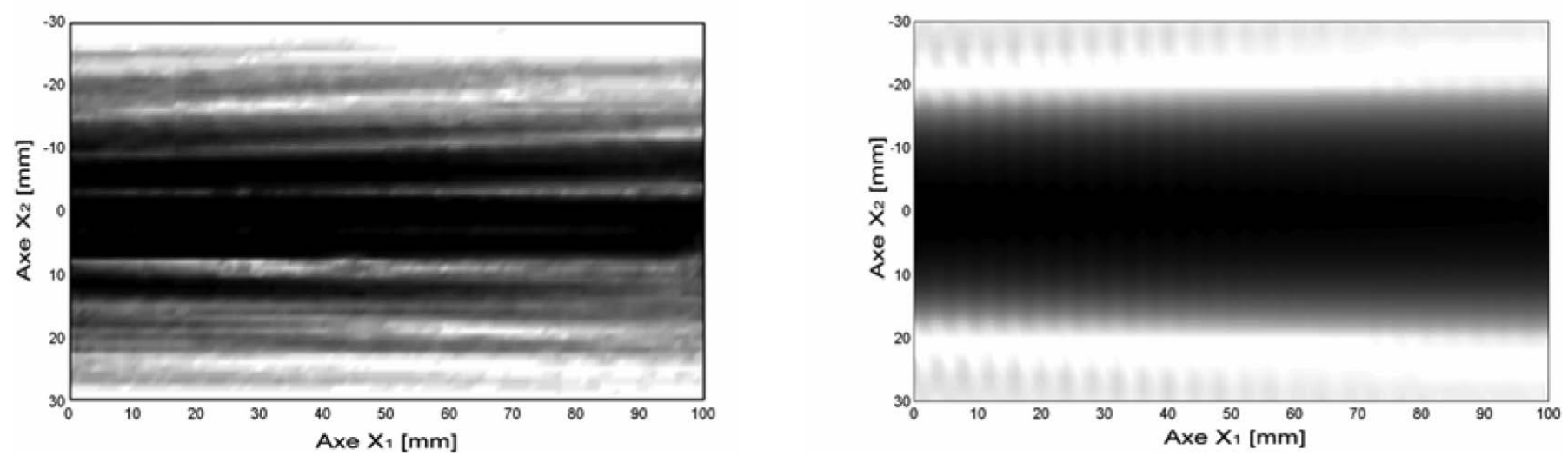

Fig. 17. Reflected field of pressure (dB). Experimental result (a) and numerical result (b). Mode $S_{0}$ excitation with an azimuthal angle $\varphi=0^{\circ}$, frequency $=1.35 \mathrm{MHz}$, incident angle $=9.8^{\circ}$, plate thickness $=0.59 \mathrm{~mm}$.
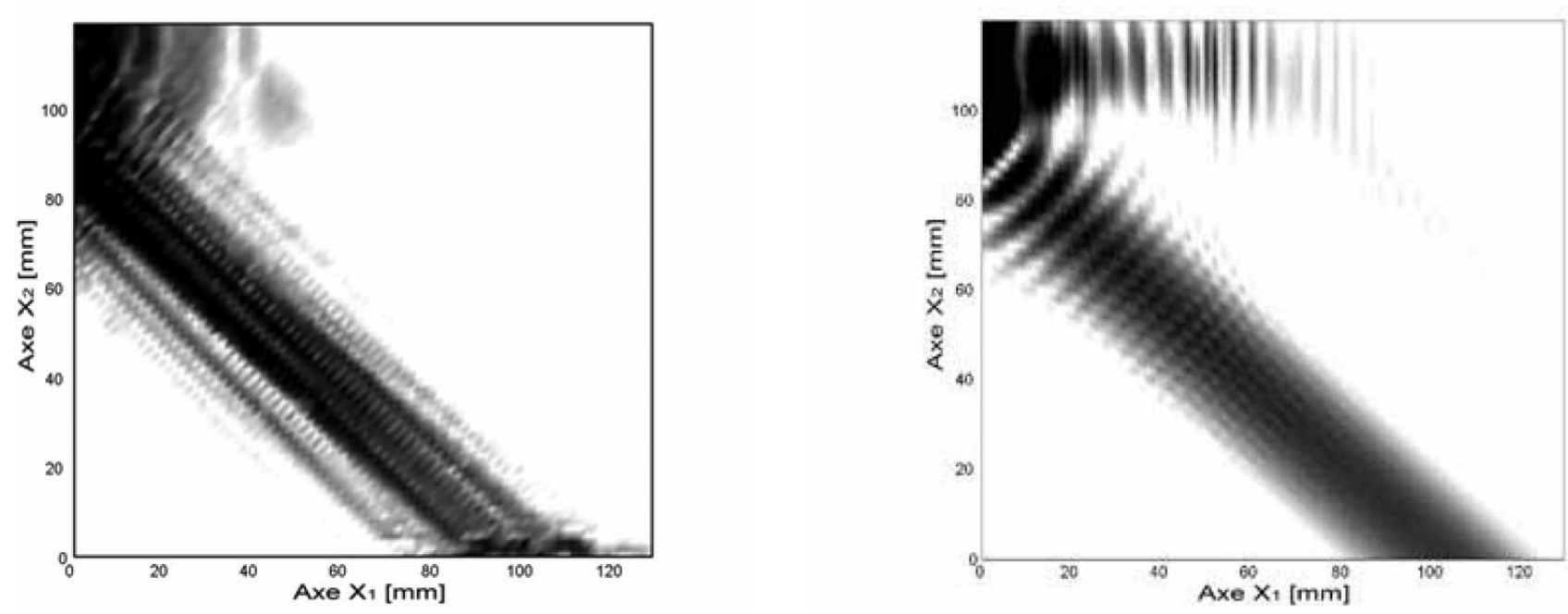

Fig. 18. Reflected field of pressure (dB). Experimental result (a) and numerical result (b). Mode $S_{0}$ excitation with an azimuthal angle $\varphi=45^{\circ}$, frequency $=1.20 \mathrm{MHz}$, incident angle $=13.6^{\circ}$, plate thickness $=0.59 \mathrm{~mm}$.

has an integrated preamplifier with a sensitivity equal to $413 \mathrm{mV} / \mathrm{MPa}$ at $3 \mathrm{MHz}$. The electrical signal is a windowed tone amplified by a Matec TB-100 electronic card (Matec Instrument Company, Northborough, MA). The duration of excitation has been adjusted in order to obtain 10 cycles within the excitation window.

The distance between the transducer emitter front face and the surface of the plate is equal to $90 \mathrm{~mm}$. The hydrophone is positioned as close as possible from the surface of the plate (roughly $1 \mathrm{~mm}$ ). It is supported by an electronically controlled arm. Displacements accuracy is $\pm 0.1 \mathrm{~mm}$ along both the $x_{1}$ and the $x_{2}$-axis (see Fig. 16).

\section{B. In Plane Configuration}

Fig. 17 presents experimental and numerical results when the fiber direction is contained in the sagittal plane (in-plane fiber configuration), i.e., $\varphi=0^{\circ}$. The excited mode is $S_{0}$. Globally, a good agreement between experi- mental and numerical shapes of the reflected pressure can be observed. The deviation angle of the Lamb beam is equal to zero in both cases; the Lamb beam propagation direction is parallel to the fiber direction and is contained in the sagittal plane.

\section{Out-of-Plane Configuration}

Fig. 18 presents experimental and numerical results when the fiber direction is not contained in the sagittal plane (out-of-plane fiber configuration). Here $\varphi=45^{\circ}$. The excited mode is still $S_{0}$. The fact that the direction of the ultrasonic reflected Lamb beam is not contained in the sagittal plane is highlighted in Fig. 18. The deviation angle obtained by seeking the maximum amplitude of the numerical and experimental reflected fields are, respectively, $42.2^{\circ}$ and $45.1^{\circ}$. Moreover, the pressure magnitudes are roughly similar. The Lamb beam propagation direction is quite close to the fiber direction. 


\section{CONCLUSiOns}

When an ultrasonic beam is incident on an anisotropic multilayered structure, the bounded nature of this beam excites a Lamb wave beam in the structure. This modal beam travels in the structure and reradiates waves into the external fluid. Due to the anisotropy of the plate, the most energetic part of the Lamb wave beam is deviated with respect to the sagittal plane. Using an asymptotic analysis, this deviation direction corresponds to the normal to the tangent of the Lamb slowness curve, at a point corresponding to the main wave number vector of the acoustic axis of the incident beam. It has been shown in this paper that a 3-D model is necessary to simulate this phenomenon. The theoretical and numerical deviation angle are in good agreement, and the oblique plane in which the Lamb wave beam reradiates in the fluid has been brought out. A very good agreement between numerical and experimental results has been found for several different configurations.

\section{REFERENCES}

[1] D. E. Chimenti and A. H. Nayfeh, "Leaky Lamb waves in fibrous composite laminates," J. Appl. Phys., vol. 58, no. 12, pp. 4531$4538,1985$.

[2] D. E. Chimenti and A. H. Nayfeh, "Anomalous ultrasonic dispersion in fluid-coupled fibrous composite plates," Appl. Phys. Lett., vol. 49, no. 9, pp. 492-493, 1986.

[3] D. E. Chimenti and A. H. Nayfeh, "Ultrasonic dispersion in fluidcoupled composite plate," in Review of Progress in Quantitative NDE. vol. 7A, D. O. Thompson and D. E. Chimenti, Eds. New York: Plenum, 1987, pp. 1085-1092.

[4] D. E. Chimenti and S. I. Rokhlin, "Relationship between leaky Lamb modes and reflection coefficient zeros for a fluid-coupled elastic layer," J. Acoust. Soc. Amer., vol. 88, pp. 1603-1611, 1990.

[5] D. E. Chimenti and R. W. Martin, "Nondestructive evaluation of composite laminates by leaky Lamb waves," Ultrasonics, vol. 29, pp. 13-21, 1991.

[6] D. E. Chimenti, J. G. Zhang, S. Zeroug, and L. B. Felsen, "Interaction of acoustic beams with fluid-loaded elastic structures," $J$. Acoust. Soc. Amer., vol. 95, no. 1, pp. 45-59, 1994.

[7] D. E. Chimenti, "Guided waves in plates and their use in materials characterization," Appl. Mech. Rev., vol. 50, no. 5, pp. 247-284, 1997.

[8] O. I. Lobkis and D. E. Chimenti, "3-D Transducer voltage in anisotropic materials characterization," J. Acoust. Soc. Amer., vol. 106C, pp. 36-45, 1999.

[9] H. Zhang and D. E. Chimenti, "2-D and 3-D complextransducer-point analyses of beam reflection from anisotropic plates," J. Acoust. Soc. Amer., vol. 108, pp. 2729-2737, 2000.

[10] N. Guo and P. Cawley, "The interaction of Lamb waves with delaminations in composite laminates," J. Acoust. Soc. Amer., vol. 94, pp. 2240-2246, 1993.

[11] T. Kundu, "On the nonspecular reflection of bounded beams," $J$. Acoust. Soc. Amer., vol. 83, pp. 19-24, 1988.

[12] T. Kundu, C. Potel, and J. F. de Belleval, "Importance of the near Lamb mode imaging of multilayered composite plates," Ultrasonics, vol. 39, pp. 283-290, 2001.

[13] A. U. Rehman, C. Potel, and J. F. de Belleval, "Numerical modeling of the effects on reflected acoustic field for the changes in internal layer orientation of a composite," Ultrasonics, vol. 36, pp. 343-348, 1998.

[14] G. Neau, M. Deschamps, and M. J. S. Lowe, "Group velocity of Lamb waves in anisotropic plates: Comparison between theory and experiment," in Review of Progress in Quantitative NDE. vol. 20, D. O. Thompson and D. E. Chimenti, Eds. New York: American Institute of Physics, 2001, pp. 81-88.

[15] G. Neau, M. J. S. Lowe, and M. Deschamps, "Propagation of Lamb waves in anisotropic and absorbing plates: Theoretical derivation and experiments," in Review of Progress in Quantitative NDE. vol. 21, D. O. Thompson and D. E. Chimenti, Eds. New York: American Institute of Physics, 2002, pp. 1062-1069.

[16] R. P. Dalton, P. Cawley, and M. J. S. Lowe, "The potential of guided waves for monitoring large areas of metallic aircraft fuselage structure," J. Nondestr. Eval., vol. 20, pp. 29-46, 2001.

[17] M. R. Gorman, "Plate wave acoustic emission," J. Acoust. Soc. Amer., vol. 90, pp. 358-364, 1991.

[18] O. Poncelet, M. Deschamps, and M. Lowe, "Célérité de groupe des ondes de Lamb dans une plaque anisotrope," in Proc. Fifth French Conf. Acoust., Sep. 2000, pp. 169-172. (in French)

[19] C. Potel, P. Gatignol, and J. F. de Belleval, "Deviation of the modal waves excited by an ultrasonic monochromatic beam in an anisotropic layer," C. R. Acad. Sci. Paris. Mec. Phys. Astr., vol. 329 , no. 11 , pp. 815-822, 2001.

[20] C. Potel, P. Gatignol, and J. F. de Belleval, "A stationary phase argument for the modal wave beam deviation in the time-space domain for anisotropic," Ultrasonics, vol. 40, pp. 549-553, 2000.

[21] S. Baly, C. Potel, J. F. de Belleval, and M. Lowe, "Numerical and experimental deviation of monochromatic Lamb wave beam for anisotropic multilayered media," in Review of Progress in Quantitative NDE. vol. 21, D. O. Thompson and D. E. Chimenti, Eds. New York: Plenum, 2001, pp. 270-277.

[22] T. D. K. Ngoc and W. G. Mayer, "A general description of ultrasonic nonspecular reflection and transmission effects for layered media," IEEE Trans. Ultrason., Ferroelect., Freq. Contr., vol. 27, pp. 229-236, 1980.

[23] J. Pott and J. G. Harris, "Scattering of an acoustic Gaussian beam from a fluid-solid interface," J. Acoust. Soc. Amer., vol. 76, no. 6, pp. 1829-1837, 1984.

[24] J. G. Harris and J. Pott, "Further studies of the scattering of a Gaussian beam from a fluid-solid interface," J. Acoust. Soc. Amer., vol. 78, no. 3, pp. 1072-1080, 1985.

[25] W. G. Neubauer, "Ultrasonic reflection of a bounded beam at Rayleigh and critical angles for a plane liquid/solid interface," $J$. Appl. Phys., vol. 44, pp. 48-53, 1973.

[26] H. L. Bertoni and T. Tamir, "Unified theory of Rayleigh-angle phenomena for acoustic beams at liquid-solid interfaces," $J$. Appl. Phys., vol. 5, pp. 157-172, 1973.

[27] H. L. Bertoni and T. Tamir, "Reflection phenomena for acoustic beams incident on a solid at a Rayleigh angle," in Proc. IEEE Ultrason. Symp., 1979, pp. 153-156, (collected papers on Nondestructive Eval. Indus. Appl.), (reprinted from 1973 Ultrason. Symp. Proc.).

[28] M. Rousseau and P. Gatignol, "Asymptotic analysis of nonspecular effects for the reflection and transmission of Gaussian beam incident on a solid plate," J. Acoust. Soc. Amer., vol. 80, pp. 325-332, 1985.

[29] M. Rousseau and P. Gatignol, "Theory of the acoustic bounded beam," in Acoustic Interactions with Submerged Elastic Structures. pt. I, vol. 5, A. Guran, J. Ripoche, and F. Ziegler, Eds. Singapore: World Scientific Publishing Co. Ltd., 1996, pp. 207241, (Series on stability, vibration and control of systems Series B).

[30] M. Rousseau and P. Gatignol, "Short wave analysis of nonspecular effects for the reflection and transmission of a Gaussian acoustic beam incident on a solid plate," J. Acoust. Soc. Amer., vol. 80, pp. 325-332, 1985.

[31] T. E. Matikas, M. Rousseau, and P. Gatignol, "Experimental studies of focusing ultrasonic beams reflected on a fluid-solid interface in the neighborhood of the Rayleigh incidence," IEEE Trans. Ultrason., Ferroelect., Freq. Contr., vol. 39, no. 6, pp. 737-744, 1992.

[32] T. E. Matikas, M. Rousseau, and P. Gatignol, "Theoretical analysis for the reflection of a focused ultrasonic beam from a fluidsolid interface," J. Acoust. Soc. Amer., vol. 93, no. 3, pp. 14071416, 1993.

[33] K. W. Ng, T. D. K. Ngoc, J. A. McClure, and W. G. Mayer, "Nonspecular transmission effects for ultrasonic beams incident on a solid plate in a liquid," Acustica, vol. 48, pp. 168-173, 1981.

[34] J. M. Claeys and O. Leroy, "Reflection and transmission of bounded beams on halfspaces and through plates," J. Acoust. Soc. Amer., vol. 72, pp. 585-590, 1982.

[35] G. Quentin, A. Derem, and B. Poirée, "The formalism of evanescent plane waves and its importance in the study of the generalized Rayleigh wave," J. d'Acoustique, vol. 3, no. 4, pp. 321-336, 1990. 
[36] J. W. Goodman, Introduction to Fourier Optics. New York: McGraw-Hill, 1981.

[37] B. Hosten and M. Deschamps, "Transmission ultrasonore en faisceau borné d'une interface plane à l'aide du spectre angulaire d'ondes planes," Traitement du Signal, vol. 2, pp. 195-199, 1985.

[38] M. E. Schaffer, P. A. Lewin, and J. M. Reid, "Propagation through inhomogeneous media using the angular spectrum method," in Proc. IEEE Ultrason. Symp., 1987, pp. 943-945.

[39] M. E. Schaffer and P. A. Lewin, "Transducer characterization using the angular spectrum method," J. Acoust. Soc. Amer., vol. 85, pp. 2204-2214, 1989.

[40] A. Souissi, "Développement des méthodes numériques et expérimentales pour l'étude du champ acoustique de transducteurs ultrasonores en présence d'une interface fluide-solide (réflexion-transmission)," Ph.D. thesis, Université de Technologie de Compiègne, Compiègne, France, 1987. (in French)

[41] J. F. de Belleval, A. Souissi, P. Gatignol, and N. Mercier, "Modélisation numérique du champ acoustique de transducteurs en présence d'interfaces quelconques ou de milieux multicouches," in Proc. 9th J. d'Etudes Sur la Propagation Acoustique. vol. 51, no. 17, 1990, pp. 33-41. (in French)

[42] D. Orofino and P. Pedersen, "Angle-dependent spectral distortion for an infinite planar fluid-fluid interface," J. Acoust. Soc. Amer., vol. 92, pp. 2883-2889, 1992.

[43] D. Orofino and P. Pedersen, "An angular spectrum technique for calculating receiver output signals for pulse-echo ultrasound insonification of elastics plates," in Proc. IEEE Ultrason. Symp., 1992, pp. 667-670.

[44] D. Orofino and P. Pedersen, "Efficient angular spectrum decomposition of acoustic source. Part I: Theory," IEEE Trans. Ultrason., Ferroelect., Freq. Contr., vol. 40, pp. 238-249, 1993.

[45] D. Orofino and P. Pedersen, "Efficient angular spectrum decomposition of acoustic sources. Part II: Results," IEEE Trans. Ultrason., Ferroelect., Freq. Contr., vol. 40, pp. 250-257, 1993.

[46] D. Orofino and P. Pedersen, "Evaluation of IP-ASD for elastic media via angular spectrum decomposition," J. Acoust. Soc. Amer., vol. 93, no. 3, pp. 1235-1248, 1993.

[47] D. Orofino and P. Pedersen, "Evaluation of angle dependent spectral distortion for infinite, planar elastic media via angular spectrum decomposition," J. Acoust. Soc. Amer., vol. 93, no. 3, pp. 1235-1247, 1993.

[48] P. Pedersen and D. Orofino, "Modeling of received ultrasound signals from finite planar targets," IEEE Trans. Ultrason., Ferroelect., Freq. Contr., vol. 43, pp. 303-311, 1996.

[49] S. Zeroug and L. B. Felsen, "Nonspecular reflection of two- and three-dimensional acoustic beams from fluid-immersed planelayered elastic structures," J. Acoust. Soc. Amer., vol. 95, pp. 3075-3089, 1994.

[50] A. U. Rehman, "Interaction d'un faisceau ultrasonore avec un milieu multicouche anisotrope à l'aide de la décomposition en spectre angulaire," Ph.D. thesis, Université de Technologie de Compiègne, France, Compiègne, France, 1998. (in French)

[51] A. U. Rehman, C. Potel, and J. F. de Belleval, "Numerical modeling of the effects on reflected acoustic field for the changes in internal layer orientation of a composite," Ultrasonics, vol. 36, no. 1-5, pp. 343-348, 1998.

[52] B. A. Auld, Acoustic Fields and Waves in Solid. New York: Wiley, 1973.

[53] D. Royer and E. Dieulesaint, Elastic Waves in Solids I: Free and Guided Propagation. Berlin: Springer-Verlag, 2000.

[54] B. Pavlakovic, M. Lowe, D. Alleyne, and P. Cawley, "Disperse: A general purpose program for creating dispersion curves," in Review of Progress in Quantitative NDE. vol. 16, D. O. Thompson and D. E. Chimenti, Eds. New York: Plenum, 1997, pp. 185-192.

[55] T. Lhermitte and B. Perrin, "Dispersion relations of elastic shear waves in cross-ply fiber reinforced composites," in Proc. IEEE Ultrason. Symp., 1991, pp. 825-830.

[56] W. T. Thomson, "Transmission of elastic waves through a stratified solid medium," J. Appl. Phys., vol. 21, pp. 89-93, 1950.

[57] N. A. Haskell, "The dispersion of surface waves in multilayered media," Bull. Seismol. Soc. Amer., vol. 43, pp. 17-34, 1953.

[58] C. Potel and J. F. de Belleval, "Acoustic propagation in anisotropic periodically multilayered media: A method to solve numerical instabilities," J. Appl. Phys., vol. 74, no. 4, pp. 22082215, 1993.
[59] M. J. S. Lowe, "Matrix techniques for modeling ultrasonic waves in multilayered media," IEEE Trans. Ultrason., Ferroelect., Freq. Contr., vol. 42, pp. 525-542, 1995.

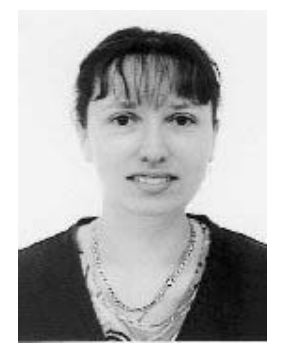

Catherine Potel received a Ph.D. degree in mechanical engineering from the University of Technology of Compiègne (UTC), Compiègne, France, in 1994. She was lecturer at the Université de Picardie Jules Verne, Amiens, France, from 1996 to 2001, and is now Professor of Physical Acoustics and of Mechanics at the Université du Maine, Le Mans, France, in the Laboratoire d'Acoustique de l'Université du Maine, since 2001.

Her research is in ultrasonics for nondestructive evaluation and materials characterization, with special interest in propagation in anisotropic multilayered media such as composites.

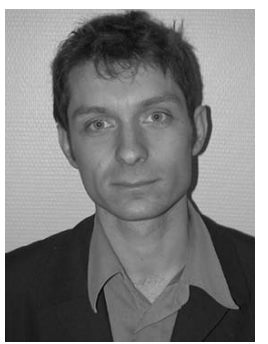

Stephane Baly received the mechanical engineering degree in 1995 and the Ph.D. degree in 2003 from the Universite de Technologie de Compiègne, Compiègne, France.

Since February 2003, he has worked in the Department of Mechanical Engineering at Hautes Etudes Industrielles at Lille, France, and is currently a lecturer. His field of interest is nondestructive evaluation (NDE), with special interest in the use of structureguided ultrasonic waves for the inspection of anisotropic structures.

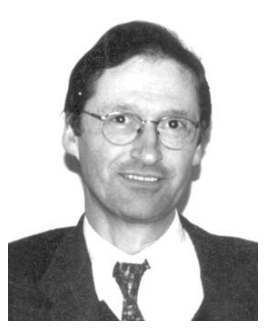

Jean François de Belleval (M'88) was born in Marseille, France. He received the diploma of engineer at the "Ecole Polytechnique", Paris and the degree of "Thèse d'Etat" (Ph.D.) of the University of Paris VI in 1974, on the subject of the relationship between the acoustic field of a hot jet and its turbulence and infrared emission.

He has been a Professor of Physical Acoustics at the University of Technology of Compiègne (UTC), Compiègne, France, since 1976. He was Director of the "Laboratoire Roberval", research unit associated at the French National Center for Scientific Research (CNRS) from 1992 to 2003. Previously he worked at ONERA (French Aerospace Research Center).

His research is in ultrasonics for nondestructive evaluation and materials characterization with special interest for propagation in anisotropic multilayered media such as composites.

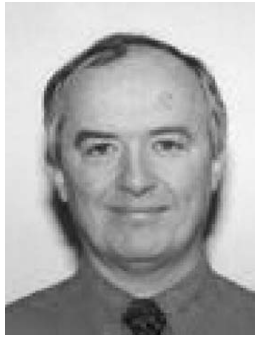

Michael J. S. Lowe received the B.Sc. degree in civil engineering from the University of Edinburgh, Edinburgh, Scotland, UK, in 1979, and the M.Sc. and Ph.D. degrees in mechanical engineering from Imperial College, University of London, in 1987 and 1993, respectively.

From 1979 to 1989 he worked in engineering consultancy, specializing in the application and development of numerical methods for the solution of problems in solid mechanics. His principal clients were in the nuclear power and offshore oil industries. Since 1989 he has worked in the Department of Mechanical Engineering at Imperial College, University 
of London, and is currently a reader. His research is in nondestructive evaluation (NDE), with special interest in the use of structure-guided ultrasonic waves for the inspection of structures. His work covers both fundamental theoretical and modeling developments, and practical transfer of findings to industrial application. He has published about 130 papers on NDE and related fields.

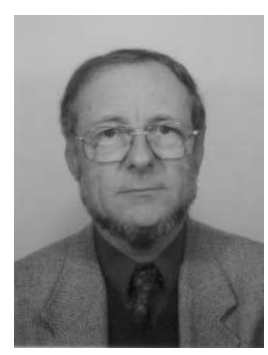

Philippe Gatignol is holder of the agrégation in the field of mathematics. He received the degree of "Thèse d'Etat" (Ph.D.) of the University of Paris VI in 1978. He was lecturer at the University of Paris VI from 1962 to 1981. He now has been Professor of Physical Acoustics at the University of Technology of Compiègne (UTC), Compiègne, France, since 1981. He was project leader at the Scientific Direction of the Department Sciences for Engineering at the National Scientific Research Center (CNRS), Paris, France, and at the French Ministry of Research, Paris, France, respectively, from 1986 to 1992 , and from 1998 to 2000 . He was also responsible for the training of Ph.D. students in mechanics at UTC until 2004. His research is in physical acoustics and ultrasonics for nondestructive evaluation. 